\title{
Effective Data Transmission and Control Based on Social Communication in Social Opportunistic Complex Networks
}

\author{
Weiyu Yang, Jia Wu $\mathbb{D}$, and Jingwen Luo \\ School of Computer Science and Engineering, Central South University, Changsha 410083, China \\ Correspondence should be addressed to Jia Wu; jiawu5110@163.com
}

Received 7 March 2020; Revised 26 April 2020; Accepted 6 May 2020; Published 8 June 2020

Guest Editor: Fei Xiong

Copyright (C) 2020 Weiyu Yang et al. This is an open access article distributed under the Creative Commons Attribution License, which permits unrestricted use, distribution, and reproduction in any medium, provided the original work is properly cited.

\begin{abstract}
In opportunistic complex networks, information transmission between nodes is inevitable through broadcast. The purpose of broadcasting is to distribute data from source nodes to all nodes in the network. In opportunistic complex networks, it is mainly used for routing discovery and releasing important notifications. However, when a large number of nodes in the opportunistic complex networks are transmitting information at the same time, signal interference will inevitably occur. Therefore, we propose a low-latency broadcast algorithm for opportunistic complex networks based on successive interference cancellation techniques to improve propagation delay. With this kind of algorithm, when the social network is broadcasting, this algorithm analyzes whether the conditions for successive interference cancellation are satisfied between the broadcast links on the assigned transmission time slice. If the conditions are met, they are scheduled at the same time slice, and interference avoidance scheduling is performed when conditions are not met. Through comparison experiments with other classic algorithms of opportunistic complex networks, this method has outstanding performance in reducing energy consumption and improving information transmission efficiency.
\end{abstract}

\section{Introduction}

With the development of mobile communications in recent years, opportunistic complex networks appear in people's vision. The difference between opportunistic complex networks and the traditional mesh network mainly lying in their nodes deployment is not uniform, the network size and initial location of the nodes are not set in advance, and there is no need for a complete path between the source node and the target node [1-4]. As nodes move, two nodes can enter the communication range and exchange data [5]. Opportunistic complex networks can handle problems that are difficult to solve with existing wireless technology networks, such as network splits and delays, and can meet the network communication that requires low-cost and terrible conditions $[6,7]$. In fact, part of the concept of opportunistic complex networks is derived from the early research on delay tolerant network (DTN) [8]. At present, the main application fields of opportunistic complex networks include wildlife tracking, handheld device networking, in-vehicle network, and remote area network transmission $[9,10]$.
Nowadays, with the advent of the $5 \mathrm{G}$ era, mobile devices such as smart phones, Bluetooth, and tablet computers have significantly increased and are widely distributed in various regions. Because people always move with these mobile devices, these devices have random mobility $[11,12]$. In this way, they can be regarded as a social node, and the community can be established through the relationship between the nodes $[13,14]$. However, when data are transmitted from the source node to other nodes in the network through broadcast, in many cases, it is desirable that the broadcast delay can be as low as possible [7]. Therefore, research on a low-latency broadcast algorithm is of great theoretical significance and practical application value for the study of information transmission in opportunistic complex networks $[15,16]$.

There are many nodes in the opportunistic complex networks. In this way, when large-scale communication occurs in a social network, many nodes in the network will simultaneously spread information [17]. So, due to the broadcast characteristics of wireless signal transmission, the signal transmission of a node will affect the data acceptance 
of other nodes within its interference range, and signal interference is an important factor that affects the node broadcast delay [18]. The above situation will result in low transmission rate and high latency for social networks. However, it has been proved [19] that, due to the influence of signal interference, the minimum delay broadcasting problem in wireless sensor networks is NP (nonpolynomial) difficult problem, so it is difficult to design a polynomial time optimization algorithm. In order to solve the problem of low-latency broadcasting in opportunistic complex networks, researchers have proposed many approximation algorithms. The broadcast delay is continuously optimized by approximating optimization to approximate the performance of the optimal algorithm [20-22]. Through these studies, the approximate ratio is continuously reduced and the performance is continuously improved. The link scheduling strategy designed under the physical interference model can effectively improve the actual performance of the broadcast algorithm $[23,24]$. However, it is necessary to consider all data links transmitting simultaneously, which makes the problem more complicated and difficult, and the research challenges are greater [25].

However, the abovementioned research work has designed the broadcast algorithm through interference avoidance scheduling technology [26]. That is, when there is a signal interference between broadcast links, the transmission of information between these nodes is distributed to different time slices to avoid mutual interference. Although the interference avoidance scheduling technology can effectively reduce the interference between signals, it reduces the number of broadcast links that can be transmitted concurrently, which is not conducive to reducing the broadcast delay. In this paper, a greedy broadcast algorithm GreedyA (greedy algorithm) is first proposed. This algorithm mainly uses the breadth first search tree with the source node as the root node to stratify the nodes in the social network [27, 28]. Then GreedyA algorithm according to the rules that the node covering more number of nodes have prioritize to become the parent node to construct the broadcast tree. Finally, the broadcast link scheduling is carried out around the broadcast tree using the idea of layerby-layer scheduling and interference avoidance scheduling. So as to better enhance the transmission performance, this paper proposes a broadcast algorithm (EDTC) based on the GreedyA algorithm, which increases the number of broadcast links that can be transmitted concurrently through successive interference cancellation techniques [29-31].

The main contributions of this research are as follows:

(1) A greedy broadcast algorithm, GreedyA. This algorithm uses the method of layer-by-layer scheduling and interference avoidance scheduling to allocate the transmission time slices of the broadcast link, which effectively solves the problem of signal interference. But the number of broadcast links that can be transmitted concurrently is limited.

(2) Combining with successive interference cancellation techniques, another low-latency broadcast algorithm
EDTC is proposed. This algorithm is based on the greedy broadcast algorithm GreedyA and makes full use of the benefits of successive interference cancellation to perform broadcast link scheduling. The EDTC algorithm improves the performance of information transmission in opportunistic complex networks by relaxing the link interference limit and increasing the number of broadcast links that can be transmitted concurrently.

(3) Experiments show that using the EDTC algorithm for information transmission in opportunistic complex networks has excellent performance in reducing energy consumption and improving data transmission efficiency.

This paper is divided into five parts. The first part introduces our research. In the second part, we briefly explained the related work. The third part introduces the proposed algorithm model. In the fourth part, a simulation experiment is performed using the proposed algorithm, and the experimental results are analyzed. Section five summarizes this study.

\section{Related Work}

Over the years, research on routing algorithms has always been a hot issue in opportunistic complex networks. So far, many routing algorithms have been proposed. Among them, there are many algorithms applied to opportunistic complex networks. Several routing algorithms are described below.

Vahdat and Becker [32] proposed the epidemic routing algorithm, whose core idea is using several meeting nodes to transmit information. Lenando and Alrfaay [33] studied epidemic with social features to improve routing performance in opportunistic social networks. The core idea of this algorithm is to utilize the social activities of the nodes. Compared with the epidemic protocol, it can increase the transmission rate and reduce the transmission overhead, average delay, and average hops. Mundur et al. [34] proposed an improvement based on epidemic routing protocols, and its core idea is to use the information that the messages have been transmitted in the antiinterference list to prevent future exchange of these messages. With this technology, there will be better buffers and network utilization, which can increase the percentage of messages delivered with a lower latency. To enhance the epidemic routing in delay-tolerant networks from an energy perspective, Rango et al. [35] proposed a new strategy to dynamically adjust the $n$-parameter. This strategy considers the energy consumption and node degree of mobile nodes to increase or decrease the amount of data distributed in the network. With this strategy, when the remaining energy of the node is low, the scalability of the popular strategy is greatly improved, and the $n$-parameter is also improved. Conversely, when the mobile node has a good energy budget, more transmissions can be allowed and the $n$-parameter can be reduced to increase the transmission probability.

Spyropoulos et al. [36] proposed a simple solution called Spray and Wait, which managed to overcome the shortcomings of epidemic routing and other flooding-based schemes. The algorithm can avoid the performance dilemma inherent in 
utility-based schemes. In order to avoid the Spray and Wait algorithm making random and blind forwarding decisions in delay tolerant networks, Xue et al. [37] proposed a Spray and Wait algorithm based on average transfer probability in delay tolerant networks. The core idea of the algorithm is using pass prediction to forward messages. Huang et al. [38] proposed a Spray and Wait routing based on location prediction in social networks. The main idea of the algorithm is that, in the waiting phase, each relay node uses polynomial interpolation to predict the future position. A copy of the message can be forwarded to another relay node closer to the target without waiting for the target node to be encountered. This solution makes full use of mobility information so that messages can be delivered to their destination faster. Jain et al. [39] proposed enhanced fuzzy logicbased Spray and Wait routing protocol for delay tolerant networks. The core idea of this algorithm is to achieve a high transfer rate by appropriately aggregating multiple message parameters. Experiments prove that compared with other Spray and Wait routing protocol variants, the proposed buffer management scheme successfully achieves the goal of increasing the delivery ratio and the overhead ratio.

For flooding routing strategies, multiple copies generated by the original nodes are used for forwarding. The network nodes have large information redundancy and high dependence on network resources. For the purpose of reducing network resources consumption to a greater extent, a routing strategy based on prediction is proposed. Dhurandher et al. [40] proposed a history-based routing prediction in opportunistic complex networks. The core idea of this algorithm is to use movement history to model the behavior of nodes. Markov predictors were used to make predictions and choose the best next node. Yu et al. [41] proposed a probabilistic routing algorithm based on contact time and message redundancy. This algorithm estimates the transit probability of a node based on the history of encounter information and contact time. And, by using a controlled replicating scheme, messages can be transmitted in parallel on multiple paths.

Based on the context-aware routing strategy, choosing the best transmission path through context-aware parameters obtained by intermediate nodes can greatly improve network performance. Wong [42] proposed the social relation opportunistic routing (SROR) algorithm. It is mainly based on social relations, social profiles, and social mobility patterns. The optimal relay node for routing data is calculated to maximize the delivery ratio. And they proved that the proposed algorithm can achieve the highest data transmission rate with the highest routing efficiency in the social environment. Xu et al. [43] proposed an intelligent distributed routing algorithm based on social similarity. This algorithm can use the social environment information in the network to predict the mobile attributes of network nodes through the BP neural network. This routing decision fully considers the time and space attributes of the mobile node. Through simulation experiments, in the comparison of other existing well-known algorithms, they find that their algorithm can improve the network's ability to adapt to topology changes.

However, in social networks, due to the broadcast characteristics of wireless signals, there will be interference between wireless signals, which will make receiving nodes to not receive the message correctly. The related research of low-latency broadcasting algorithms is introduced below with respect to the physical interference model.

Yu et al. [24] studied the basic communication primitives in unstructured wireless networks under the physical interference model and the method for distributing broadcast messages from multiple nodes to the entire network with minimum delay. They proved that the proposed random distributed algorithm can be completed in $O((D+n b) \log n+$ $\left.\log ^{2} n\right)$ time slices with a higher probability, where $D$ is the network diameter, $n_{b}$ is the number of nodes that need to send broadcast messages, and $n$ is the network scale.

Tian et al. [44] proposed two global broadcast distributed determination algorithms based on the signal-to-interference plus noise ratio model. In these two algorithms, any node can become the source node, and the remaining nodes are divided into different layers according to their distance from the source node. Broadcast messages are transmitted layer by layer from the source node to all other nodes. For the first algorithm, by carefully selecting multiple subsets of the largest independent set of each layer, most concurrent transmissions can be allowed. Its time complexity is $O(D \log n)$. For the second algorithm, the running time is improved by reducing the number of repeated broadcast messages in each layer, that is, eliminating redundant broadcasts in the same layer. Theoretical analysis shows that the time complexity of the second algorithm is $O(D \Delta \log n)$.

However, none of the abovementioned research works have used successive interference cancellation techniques to design low-latency broadcasting algorithms. In opportunistic complex networks, different network nodes have different signal interferences. How to choose the forwarding nodes reasonably and increase the number of links that can be transmitted concurrently is very challenging. Successive interference cancellation technology can effectively decode the required signals from the disturbing signals. As a result, network performance is improved. However, as far as we know, there is no research work to apply successive interference cancellation technology to the broadcast algorithm to reduce the broadcast delay in social opportunistic complex networks, so it is necessary to conduct in-depth research.

\section{Materials and Methods}

3.1. Network Model. This paper considers an opportunistic complex network with $m$ nodes. Each sensor node uses a halfduplex omnidirectional antenna for wireless communication, and the maximum transmission distance is the same. Based on the characteristics of node wireless communication, the network is modeled as a unit circle graph $G_{0}=\left(V_{0}, E_{0}\right)$. The set $V_{0}$ contains all nodes in the network, and the set $E_{0}$ includes all edges in the network. There is an edge between two nodes if and only if the distance between them is less than or equal to the maximum transmission distance.

Assuming that the time between the nodes is synchronized, the scheduling time is divided into several time slices of the same length. Each node can finish sending or receiving a piece of data in a time slice. This paper regards the physical 
interference model as the signal interference model. That is to say, when a node's signal to noise ratio is not lower than a certain threshold, the node can correctly decode the required signal.

3.2. Problem Definition. This paper researches the broadcasting problem of opportunistic complex networks, among which the source node needs to transmit its data to all sensor nodes at time slice 1 . When all sensor nodes receive data from the source node, the broadcast task is completed. Broadcast scheduling is used to allocate the transmission time slice of each node. The goal of this paper is to determine how to optimize the latency that all nodes receive source node data and ensure that the scheduled data transmission signals do not interfere with each other.

Definition 1. BDPIM (Broadcast Delay under Physical Interference Model) question. Given the wireless sensor network $G_{0}=\left(V_{0}, E_{0}\right)$ and a source node $j$, under the physical interference model, a broadcast algorithm is designed so that all nodes can receive data from the source node with the lowest broadcast delay.

Existing work has proved that the BDPIM problem is an NP-difficult problem [16], so it is impossible to design an optimization algorithm for polynomial time. In order to decrease the time complexity of the algorithm and to optimize the performance of the algorithm as much as possible, a low-latency broadcast algorithm with polynomial time needs to be designed.
In opportunistic complex networks, nodes use the "storage-carry-forward" routing mode to implement internode communication. When we analyze the nodes in the opportunistic complex networks, we must first know its characteristics. Therefore, we point out that all social complex network nodes meet the following conditions.

We can define that, at time $s$, the modularity of the community can be expressed as

$$
U(s)=\frac{m_{e}}{M}-\frac{l_{t}^{2}}{4 M^{2}} .
$$

Among them, $U$ is the modularity of the community. $M$ is the total weight of the edge. $m_{e}$ represents the total weight of edges in community $e . l_{t}$ expresses the total level of node $t$ in the community.

Condition 1. If the node is in an opportunistic complex network, then as the weight of edges formed with other nodes in the network increases, the total edge weight $m_{e}$ of the community will increase. The above situation will increase the relevance degree of the community in the opportunistic complex networks.

Proof. ProofAt time $s$, the modularity in the community is $U(s)$, and then, the change in modularity after time $s+1$ can be expressed as

$$
U(s+1)=\frac{m e+\Delta m}{M+\Delta m}-\frac{\left(l_{t}+2 \Delta m\right)^{2}}{4(M+\Delta m)^{2}} .
$$

Then,

$$
\begin{aligned}
U(s+1)-U(s)= & \frac{m_{e}+\Delta m}{M+\Delta m}-\frac{\left(l_{t}+2 \Delta m\right)^{2}}{4(M+\Delta m)^{2}}-\left(\frac{m_{e}}{M}-\frac{l_{t}^{2}}{4 M^{2}}\right) \\
= & \frac{\left(4 M^{3} m_{e}+4 M^{2} \Delta m m_{e}+4 M^{3} \Delta m+4 M^{2} \Delta m^{2}\right)-\left(M^{2} l_{t}^{2}+4 M^{2} \Delta m^{2}+4 M^{2} l_{t} \Delta m\right)}{4 M^{2}(M+\Delta m)^{2}} \\
& -\left(\frac{\left(4 M^{3} m_{e}+4 M \Delta m^{2} m_{e}+8 M^{2} \Delta m m_{e}\right)-\left(l_{t}^{2} M^{2}+l_{t}^{2} \Delta m^{2}+2 l_{t}^{2} M \Delta m\right)}{4 M^{2}(M+\Delta m)^{2}}\right) \\
& \geq \frac{4 M^{3} \Delta m-4 M^{2} l_{t} \Delta m-4 M^{2} m_{e} \Delta m+2 M^{2} l_{t} \Delta m}{4 M^{2}(M+\Delta m)^{2}}-\frac{4 M l_{t} \Delta m^{2}-\left(l_{t} \Delta m\right)^{2}}{2 M^{2}(M+\Delta m)^{2}} \\
& \geq \frac{4 M^{3} \Delta m-6 M^{2} l_{t} \Delta m+2 M^{2} l_{t} \Delta m-2 M^{2} l_{t} \Delta m+\left(l_{t} \Delta m\right)^{2}}{4 M^{2}(M+\Delta m)^{2}} \\
= & \Delta m \frac{4 M^{3} \Delta m-6 M^{2} l_{t}+2 M^{2} l_{t}-2 M^{2} l_{t} \Delta m+l_{t}^{2} \Delta m}{4 M^{2}(M+\Delta m)^{2}} \\
= & \Delta m \frac{\left(2 M^{2}-2 M l_{t}-l_{t} \Delta m\right) \times\left(2 M-l_{t}\right)}{4 M^{2}(M+\Delta m)^{2}}
\end{aligned}
$$

We can get $\Delta m>0$. So we just need to prove $\left(2 M^{2}-2 M l_{t}-l_{t} \Delta m\right) \times\left(2 M-l_{t}\right)>0$; then, we can get $U(s+1)-U(s)>0$.
In other words, 


$$
\begin{aligned}
& \left\{\begin{array}{l}
2 M^{2}-2 M l_{t}-l_{t} \Delta m>0, \\
2 M-l_{t}>0,
\end{array}\right. \\
& \left\{\begin{array} { l } 
{ 2 M ^ { 2 } - 2 M l _ { t } - l _ { t } \Delta m > 0 } \\
{ 2 M - l _ { t } > 0 } \\
{ \Delta m > 0 }
\end{array} \Longrightarrow \left\{\begin{array}{l}
0<\Delta m<2 M\left(\frac{M}{l_{t}}-1\right) \\
l_{t}<2 M
\end{array}\right.\right. \\
& \Longrightarrow\left\{\begin{array} { l } 
{ 0 < \Delta m < 2 M ( \frac { M } { l _ { t } } - 1 ) } \\
{ 2 M ( \frac { M } { l _ { t } } - 1 ) > 0 } \\
{ l _ { t } < 2 M }
\end{array} \Longrightarrow \left\{\begin{array}{l}
0<\Delta m<2 M\left(\frac{M}{l_{t}}-1\right), \\
l_{t}<M .
\end{array}\right.\right.
\end{aligned}
$$

However, we also know that $2 U$ is the sum degree of the nodes in the network, and no community in the network has a saturation greater than $2 U$. In summary, we can get that increasing the weight can increase the relevance to the community in the social opportunity network. That is to say, we can get that if a node belongs to this opportunistic complex networks, its weight will affect the relevance of the community in the social opportunity network.

Condition 2. In an opportunistic complex network, if node $N_{i}$ meets the condition $l_{i} l_{j} / 2 M<m_{i j}<\Delta m+\left(l_{i} l_{j}+\right.$ $\left.l_{t} \Delta m+\Delta m^{2} / 2(M+\Delta m)\right)$, then it will be separated from the community of node $C_{j}$.

Proof. We first assume that community $E$ is divided into two subcommunities $E_{i}$ and $E_{j}$, where nodes $N_{i}$ and $C_{j}$ are in different communities. The total weight of the community decreases. Then,

$$
\left\{\begin{array}{l}
M_{i}+M_{j}<M, \\
\frac{m_{i}}{M}-\frac{l_{i}^{2}}{4 M^{2}}+\frac{m_{j}}{M}-\frac{l_{j}^{2}}{4 M^{2}}<\frac{l_{i}+l_{j}+m_{i j}}{M}-\frac{\left(l_{i}+l_{j}\right)^{2}}{4 M^{2}}, \\
m_{i j}>\frac{l_{i} l_{j}}{2 M} .
\end{array}\right.
$$

When the total weight has decreased, we can express the formula as

$$
\begin{aligned}
& M_{i}^{*}+ M_{j}^{*}>M^{*}, \\
& m_{i j}<\Delta m+\frac{l_{i} l_{j}+l_{t} \Delta m+\Delta m^{2}}{2(M+\Delta m)} .
\end{aligned}
$$

As mentioned above, we can know that if two nodes in communities $E_{i}$ and $E_{j}$ satisfy the condition $l_{i} l_{j} / 2 M<m_{i j}<\Delta m+\left(l_{i} l_{j}+l_{t} \Delta m+\Delta m^{2} / 2(M+\Delta m)\right)$, then the community has been divided.

Condition 3. For node $N_{i}$ in the opportunistic network, if its edge is connected to node $C_{j}$ and this edge is the only edge of node $C_{j}$, then when the weight between nodes $N_{i}$ and $C_{j}$ drops, node $C_{j}$ will still not be separated from the community.

Proof. If community $E$ is divided, then it must meet the following three conditions:

$$
\left\{\begin{array}{l}
M_{i}+M_{j}<M, \\
\frac{u_{i}}{M}-\frac{l_{i}^{2}}{4 M^{2}}+\frac{u_{j}}{M}-\frac{l_{j}^{2}}{4 M^{2}}<\frac{l_{i}+l_{j}+m_{i j}}{M}-\frac{\left(l_{i}+l_{j}\right)^{2}}{4 M^{2}}, \\
m_{i j}>\frac{l_{i} l_{j}}{2 M} .
\end{array}\right.
$$

Along with the weight change, the formula can be expressed as

$$
\left\{\begin{array}{l}
M_{i}^{*}+M_{j}^{*}>M^{*}, \\
m_{i j}<\Delta m+\frac{l_{i} l_{j}+l_{t} \Delta m+\Delta m^{2}}{2(M+\Delta m)} .
\end{array}\right.
$$

It can be understood as

$$
\frac{l_{i} l_{j}}{2 M}<m_{i j}<\frac{l_{i}\left(l_{j}+\Delta m\right)}{2(M+\Delta m)}=\frac{l_{i} l_{j}+l_{i} \Delta m}{2(M+\Delta m)} .
$$

Because

$$
\frac{l_{i} l_{j}+l_{i} \Delta m}{2(M+\Delta m)}-\frac{l_{i} l_{j}}{2 M}=\frac{l_{i} \Delta m\left(M-l_{j}\right)}{2 M(M+\Delta m)}<0, \quad \Delta m<0,
$$

then

$$
\frac{l_{i} l_{j}+l_{i} \Delta m}{2(M+\Delta m)}<\frac{l_{i} l_{j}}{2 M} .
$$

So, in the end, we can get $l_{i} l_{j} / 2 M<m_{i j}<\Delta m+\left(l_{i} l_{j}+\right.$ $\left.l_{t} \Delta m+\Delta m^{2} / 2(M+\Delta m)\right)$ is false.

By the above proof, we can get the conclusion that, for the nodes in opportunistic complex networks, if its edge is connected to another node and this edge is the only edge of the node, then when the weight between two nodes drops, the node will still not be separated from the community.

In summary, if a node is in an opportunistic complex network, then it should meet the above conditions.

3.3. Basic Theory of Successive Interference Cancellation. For the interference characteristics of wireless signals, traditional algorithms usually adopt the idea of interference avoidance scheduling for broadcast link scheduling. Different from the traditional interference avoidance method, this paper considers adopting successive interference cancellation technology to increase the number of broadcast 
links that can be transmitted concurrently to improve the performance of information transmission. Successive interference cancellation is a multipacket receiving technology, which can decode the required data messages from the conflicting signals and thereby effectively reduce the signal interference in wireless networks. During the iterative detection of receiving nodes with successive interference cancellation, the strongest signals are decoded, while other signals are considered interference.

The condition that a signal meets the SIC at the receiving node is that its signal internet performance noise ratio (SINR) is not lower than a specific threshold. We will try to decode the signal with the strongest signal strength when it receives the conflict signal. At this time, other transmitted signals are regarded as noise. If the decoding is successful, the receiving node removes the signal. Then, the receiving node attempts to decode the strongest of the remaining signals. This process continues until all signals are extracted or decoded fails. Through this process, all the information carried in the conflict signal can be decoded gradually, and then the required information can be obtained. This process is called SIC's sequential detection feature. Obviously, the decoding of weak signals requires the successful decoding of all stronger signals as a prerequisite. In other words, in the conflict signal, the weak signal is dependent on the strong signal.

Firstly, this article shows the association between nodes in the communication domain, as shown in Figure 1. The source nodes directly transmit information to each other, and then the source nodes transmit the information to other nodes in the communication domain through broadcasting. In a communication domain, we define 1 source node and $m$ sensor nodes.

In this paper, the noise power is defined as $W_{N_{0}}$, the specific threshold value meeting successive interference cancellation is $\chi_{\text {SIC }}$, and the distance between two nodes $A_{1}$ and $B_{1}$ is $d_{A_{1} B_{1}}$. When two data links $I_{A_{1} B_{1}}$ and $I_{A_{2} B_{2}}$ transmit simultaneously, according to the constraints of successive interference cancellation techniques, whether the node $R_{1}$ can decode the signal of $S_{1}$ has the following three cases:

(1) $I_{A_{1} B_{1}}$ is independent of $I_{A_{2} B_{2}} \cdot I_{A_{1} B_{1}}$ and $I_{A_{2} B_{2}}$ are transmitted at the same time. Node $B_{1}$ can still decode the signal of $I_{A_{1} B_{1}}$ under the interference of $I_{A_{2} B_{2}}$. The following conditions must be met:

$$
\frac{W_{B_{1}}\left(A_{1}\right)}{W_{B_{1}}\left(A_{2}+W_{N_{0}}\right)} \geq \chi_{\text {SIC }} .
$$

Among them, $W_{B_{1}}\left(A_{1}\right)$ and $W_{B_{1}}\left(A_{2}\right)$ indicate the signal strength the node $B_{1}$ received from two sending nodes $A_{1}$ and $A_{2}$. Different signal fading models can obtain different received signal strengths. For the convenience of analysis, this paper uses the same signal fading model as in [24]; that is, $W_{B_{1}}\left(A_{1}\right)$ and $W_{B_{1}}\left(A_{2}\right)$ can be calculated according to the following formula:

$$
\begin{aligned}
& W_{B_{1}}\left(A_{1}\right)=\frac{W_{A_{1}}}{d_{A_{1} B_{1}}^{n}}, \\
& W_{B_{1}}\left(A_{2}\right)=\frac{W_{A_{2}}}{d_{A_{2} B_{1}}^{n}} .
\end{aligned}
$$

Among them, $W_{A_{1}}$ and $W_{A_{2}}$ represent the signal transmission power of nodes $A_{1}$ and $A_{2}$, respectively; $n$ represents the signal attenuation index, and the value ranges from 2 to 6 . By modifying equations (14) and (15), the proposed algorithm can also be extended to other signal fading models in this paper.

As shown in Figure 2, when the distance from node $A_{2}$ to node $B_{1}$ is farther than the distance from node $A_{2}$ to node $A_{1}$, the above conditions may be satisfied.

(2) $I_{A_{1} B_{1}}$ depends on $I_{A_{2} B_{2}} \cdot I_{A_{1} B_{1}}$ and $I_{A_{2} B_{2}}$ are transmitted at the same time. On node $B_{1}$, the signal of node $A_{2}$ is stronger and satisfies the successive interference cancellation conditions. Therefore, the signal of node $A_{1}$ can be used as the interference signal. Firstly, the signal of node $A_{2}$ is decoded, and then, the signal is removed, thereby decoding the signal of node $A_{1}$. The following conditions need to be met:

$$
\frac{W_{B_{1}}\left(A_{2}\right)}{\left(W_{B_{1}}\left(A_{1}\right)+W_{N_{0}}\right)} \geq \chi_{\text {SIC }} .
$$

As shown in Figure 3, when the distance from node $A_{2}$ to node $B_{1}$ is closer than the distance from node $A_{2}$ to node $A_{1}$, the above conditions may be satisfied.

$I_{A_{2} B_{2}}$ interferes with $I_{A_{1} B_{1}} . I_{A_{1} B_{1}}$ and $I_{A_{2} B_{2}}$ are transmitted at the same time. Any signal as an interference signal does not satisfy the successive interference cancellation conditions. Node $B_{1}$ cannot decode the signal of node $A_{1}$; that is, it meets the following two conditions at the same time:

$$
\begin{gathered}
\frac{W_{B_{1}}\left(A_{1}\right)}{\left(W_{B_{1}}\left(A_{2}\right)+W_{N_{0}}\right)}<\chi_{\text {SIC }}, \\
\frac{W_{B_{1}}\left(A_{2}\right)}{\left(W_{B_{1}}\left(A_{1}\right)+W_{N_{0}}\right)}<\chi_{\text {SIC }} .
\end{gathered}
$$

As shown in Figure 4, when the distance from node $A_{2}$ to node $B_{1}$ and the distance from node $A_{2}$ to node $A_{1}$ is similar, the above conditions may be satisfied.

For the first two cases, when two links are transmitting data at the same time, node $B_{1}$ can still decode the signal of node $A_{1}$. While in the third case, node $B_{1}$ cannot decode the signal of node $A_{1}$. Based on the above characteristics of successive interference cancellation techniques, this paper will specifically design different link scheduling strategies to maximize the number of data links that can be transmitted concurrently, thereby reducing broadcast delay. 

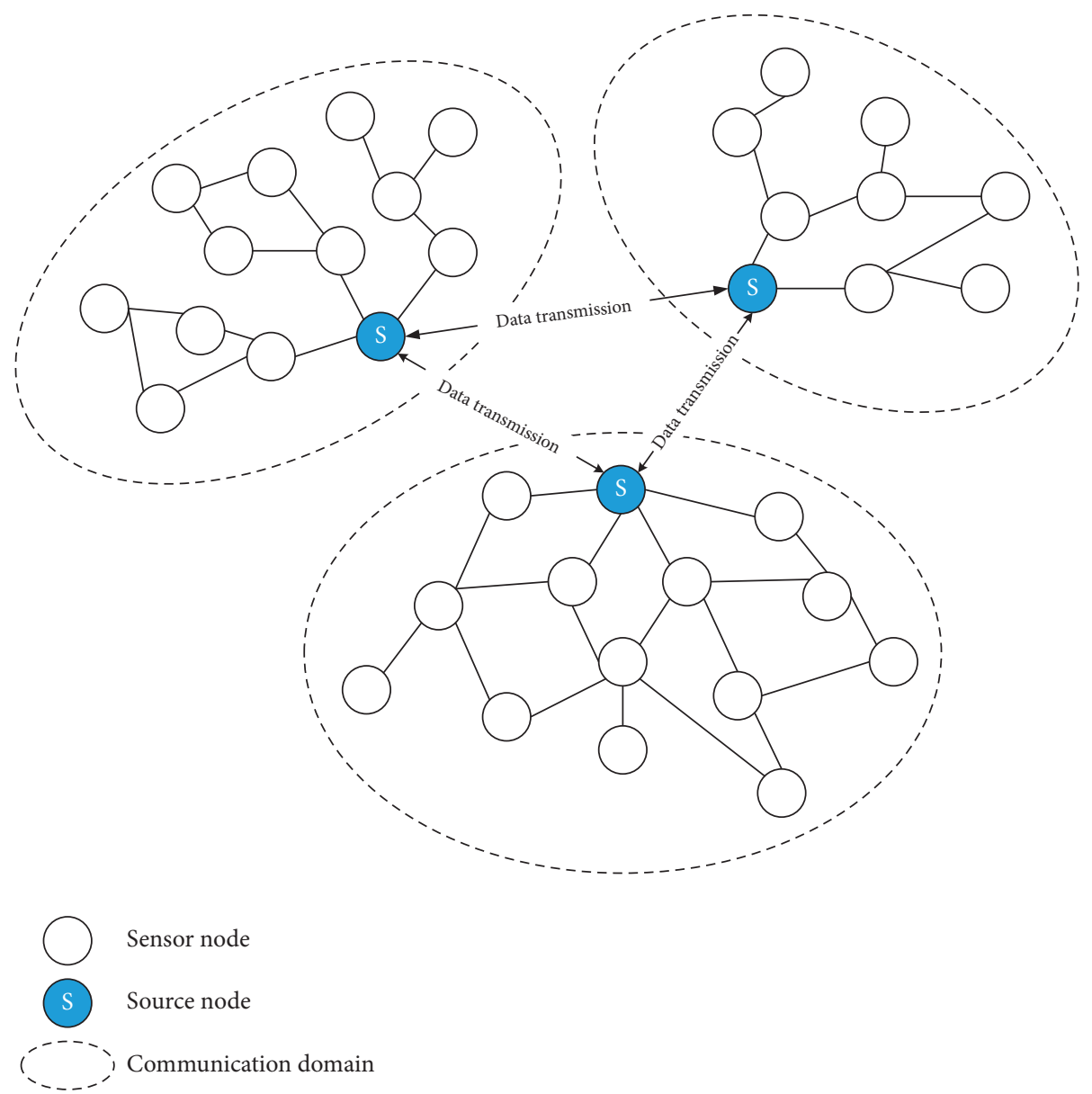

Figure 1: Data delivery on communication domain.

In [45], we can see a good application of successive interference cancellation technology in single- and multipleantenna OFDM systems. Among them, the SIC-OF system has been applied to various famous network implementations, such as cellular, ad hoc, and infrastructure-based platforms. In [46], we can see the application of SIC in uplink massive MIMO systems. In the article, they research the energy efficiency when nonlinear successive-interference cancellation (SIC) receivers are employed at the BSs and provide an asymptotic analysis of the total transmit power with zero forcing SIC. As a result, as shown by the numerical results, the EE using the SIC receiver may be significantly higher than the EE using the linear receiver.

3.4. Greedy Broadcast Algorithm. Although the theoretical delay of the greedy broadcast algorithm is usually high in the worst case, it can often obtain a good average delay in the experiment. Therefore, this paper firstly considers designing a greedy broadcast algorithm (GreedyA) in this section. Then, on the basis of this algorithm, in the next part, we design another low-latency broadcast algorithm by combining successive interference cancellation techniques. The pseudocode of the GreedyA algorithm is shown in Algorithm 1.

The GreedyA algorithm uses a layer-by-layer scheduling method for broadcast scheduling, so the first step is to construct a breadth first search tree with the source node as the root node and divide all nodes into different layers. With the purpose of effectively improving the performance of the broadcast algorithm and avoiding signal interference, the parent-child node relationship must be determined. Wireless signals have broadcast characteristics. In order to reduce the number of broadcast forwarding nodes, according to the rule that the node with the largest number of covered nodes takes precedence as the parent node, the algorithm constructs the broadcast tree $T_{s}$ layer by layer starting from the top layer.

After the broadcast tree is constructed, the broadcast scheduling is performed layer by layer from the top layer. That is, after the broadcast data transmission of the nodes in the previous layer is completed, the next layer of nodes performs broadcast data transmission. In each layer, each node with child nodes is taken out in order, and then the transmission time slice of the node is allocated. The time slice allocation method is starting from the initial scheduling time slice $t_{j}$ of this layer and analyzing whether the node has signal interference with the node that has been scheduled in the current time slice. The basis for judging the existence of signal interference is assuming that the node can perform broadcast data transmission with the node that has been scheduled in the current time slice at the same time and analyzing the receiving nodes of these nodes. If the SINR of a 


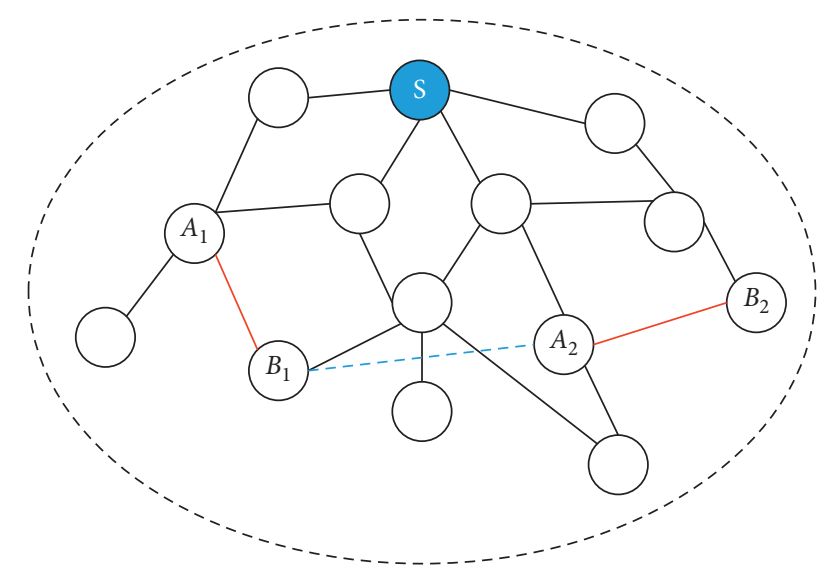

- Data transmission

- - - Separation distance

FIGURE 2: The schematic diagram that $I_{A_{1} B_{1}}$ is independent of $I_{A_{2} B_{2}}$.

receiving node is less than the specific threshold $\chi_{\text {SIC }}$, it is assumed that the assumption is not true; that is, there is signal interference between the node and the node that has been scheduled in the current time slice.

Because the GreedyA algorithm does not apply successive interference cancellation techniques, as long as the above situation occurs, the two links are considered to interfere with each other. The strategy adopted by the algorithm is performing interference avoidance scheduling, that is, dividing the interfering links into different time slices for data transmission. $M(y)$ in Algorithm 1 represents the set of all nodes whose transmission time slices are allocated in $y$ time slices, where $y$ is a positive integer. However, $|W|$ represents the number of nonempty elements $M(y)$ contained in the set $W$, and it also represents the maximum transmission time slice of all scheduled nodes.

In the following, the main idea of the GreedyA algorithm is introduced through the communication domain shown in Figure 1. First, all nodes are divided into 4 layers according to the concept of breadth first search tree. As shown in Figure 5, we can get the number of layers for each node in the communication domain.

Based on the layer of each node, we can build a breadth first search tree, as shown in Figure 6.

According to the rule that the node with the largest number of covered nodes takes precedence as the parent node, the algorithm constructs the broadcast tree $T_{s}$ layer by layer starting from the top layer $D_{0}$. For example, the number of nodes covered by node $n_{3}$ in layer $D_{1}$ is the largest, so the node $n_{3}$ is selected as the parent node of $n_{6}, n_{7}$, and $n_{8}$ in layer $D_{2}$. As shown in Figure 7, we can get the final broadcast tree. Through the idea of the GreedyA algorithm mentioned above, we list three situations that may encounter signal interference during transmission in community 1 , community 2 , and community 3 .

The next step of the GreedyA algorithm is to perform the broadcast chain scheduling. The method adopted is interference avoidance scheduling. Because there is only one

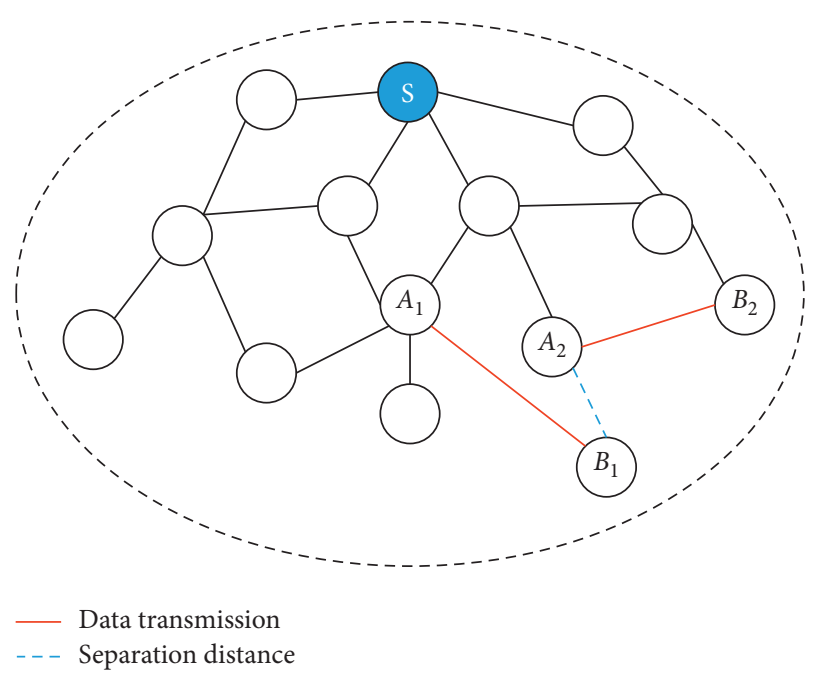

FIgURE 3: The schematic diagram that $I_{A_{1} B_{1}}$ is dependent of $I_{A_{2} B_{2}}$.

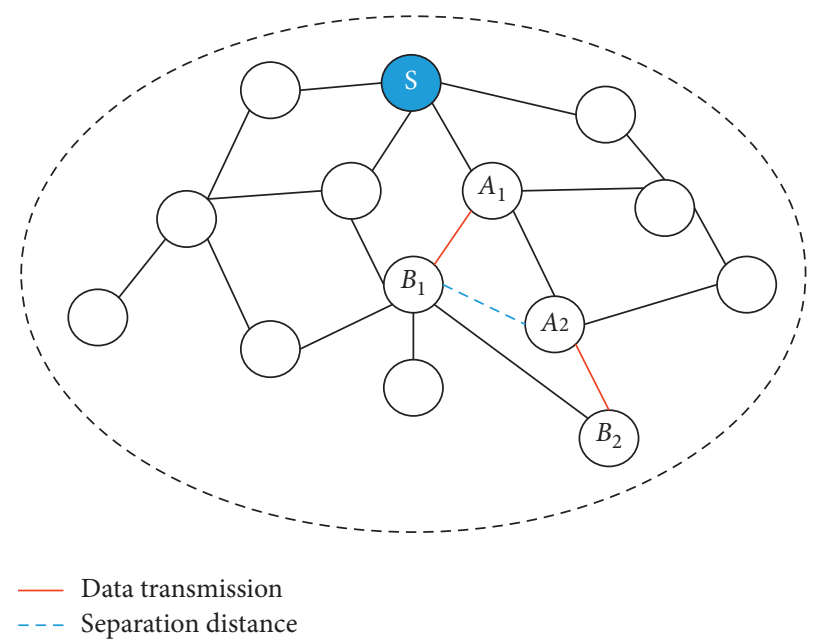

FIGURE 4: The schematic diagram that $I_{A_{1} B_{1}}$ interferes with $I_{A_{2} B_{2}}$.

sending node, scheduling in $D_{0}$ and $D_{1}$ layers is simple. In the $D_{1}$ layer, after node $n_{3}$ dispatches the transmission time slice, it needs to allocate the transmission time slice of node $n_{2}$. As shown in Figure 8, whether the signals of nodes $n_{3}$ and $n_{2}$ will affect their respective receiving nodes is analyzed; that is, whether the SINR of their receiving nodes will be below a specific threshold. If not affected, nodes $n_{3}$ and $n_{2}$ can be arranged for transmission at the same time slice; otherwise, node $n_{2}$ will be arranged for transmission at the next time slice to avoid signal interference.

In the $D_{2}$ layer, after node $n_{6}$ dispatches the transmission time slice, it needs to allocate the transmission time slice of nodes $n_{5}$ and $n_{7}$. As shown in Figure 9, first of all, it is analyzed whether the signals of nodes $n_{6}$ and $n_{5}$ will affect their respective receiving nodes, that is, whether the SINR of their receiving nodes will be below a specific threshold. If not affected, nodes $n_{6}$ and $n_{5}$ can be arranged for transmission at the same time slice; otherwise, node $n_{5}$ will be arranged for transmission at the next time slice to avoid signal interference. It is analyzed whether the signals of nodes $n_{6}$ and $n_{7}$ 
Input: network size, source node, node position, maximum transmission distance, signal transmission power, SIC-specific threshold, noise power, signal attenuation index;

Output: transmission time slice and broadcast delay of all nodes;

Begin

Let breadth first search tree $T$; //creating a breadth first search tree with the source node as the root node $T$

$D_{0}, D_{1}, D_{2}, \ldots, D_{Y} ; / /$ dividing all nodes into different layers, including $D_{0}, D_{1}, D_{2}, \ldots, D_{Y}$

$Y$;//represents the height of the breadth first search tree;

Broadcast tree $T_{S}$; //according to the rule that the node with the largest number of covered nodes takes precedence as the parent node, construct the broadcast tree $T_{S}$ layer by layer starting from the top layer $D_{0}$;

$t_{j} \longleftarrow 1 ; / /$ scheduling time slice

$M \longleftarrow \varnothing ; / /$ the set of nodes that have scheduled transmission time slices

FOR $m \longleftarrow 0$ to $Y-1 \mathrm{DO}$

FOR $n \longleftarrow 1$ to length $\left(D_{m}\right)$ DO

Take out the $n$ - th node $D_{m}(n)$ in $D_{m}$;

IF the set of child node of node $D_{m}(n)$ is not an empty set THEN

$y \longleftarrow t_{j}$;

WHILE exist signal interference between nodes $D_{m}(n)$ and $M(y)$ DO

$y \longleftarrow y+1$

END

The transmission time slice of the scheduling node $D_{m}(n)$ is $y$;

$$
M(y) \longleftarrow M(k) \cup\left\{D_{m}(n)\right\}
$$

END

$t_{j} \longleftarrow|W|+1$

Broadcast delay $\longleftarrow t_{j}$;

RETURN transmission time slice and broadcast delay of all nodes

Algorithm 1: GreedyA.

will affect their respective receiving nodes. If not affected, nodes $n_{6}$ and $n_{7}$ can be arranged for transmission at the same time slice; otherwise, node $n_{7}$ will be arranged for transmission at the next time slice to avoid signal interference. The transit time relationship between nodes $n_{5}$ and $n_{7}$ is also analyzed as above.

3.5. Broadcast Algorithm Based on Successive Interference Cancellation. It is worth noting that the method adopted by the GreedyA algorithm in broadcasting chain scheduling is the idea of interference avoiding scheduling. However, combined with successive interference cancellation technology can increase the number of broadcast links that can be transmitted concurrently in a certain extent. In this section, designing another low-latency broadcast algorithm EDTC based on successive interference cancellation technology and GreedyA algorithm is to further improve transmission performance. The steps of the EDTC algorithm and the GreedyA algorithm are basically the same, but the main difference is that the basis for judging whether the broadcast link interferes; that is, the 10th line in the pseudocode is different. The two broadcast links, $I_{A_{1} B_{1}}$ and $I_{A_{2} B_{2}}$, do not interfere with each other, and the judgment basis of the GreedyA algorithm is as follows:

result $==\left(I_{A_{1} B_{1}}\right.$ independent of $\left.I_{A_{2} B_{2}}\right) \cap\left(I_{A_{2} B_{2}}\right.$ independent of $\left.I_{A_{1} B_{1}}\right)$.
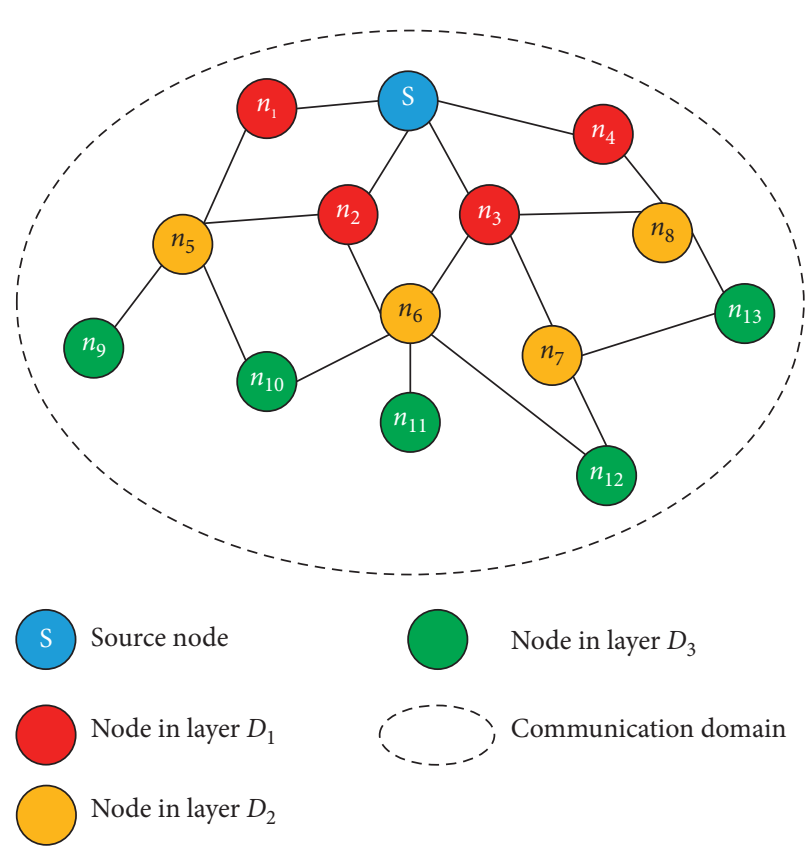

Node in layer $D_{3}$

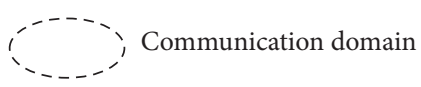

Figure 5: Node hierarchy graph.

Formula (7) requires that the SINR of the receiving nodes of the two links must be higher than the specific threshold. However, the judgment of the EDTC algorithm is 


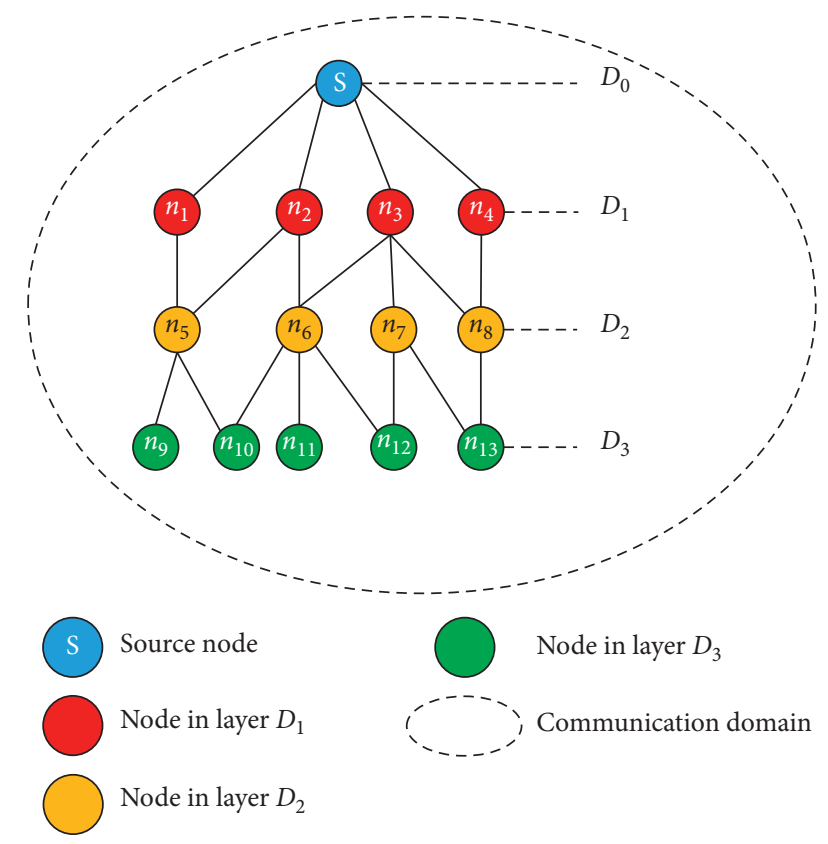

FIGURE 6: The layering of network node.

based on the interference between the two links under the SIC condition, which satisfies

$$
\text { result }=\left(I_{A_{1} B_{1}} \text { interfere } I_{A_{2} B_{2}}\right) \cap\left(I_{A_{2} B_{2}} \text { interfere } I_{A_{1} B_{1}}\right) \text {. }
$$

Among them, result is the judgment consequent. It is different from GreedyA algorithm, when $I_{A_{1} B_{1}}$ is independent of $I_{A_{2} B_{2}}$ or $I_{A_{1} B_{1}}$ depends on $I_{A_{2} B_{2}}$, EDTC algorithm considers that $I_{A_{1} B_{1}}$ does not interfere with $I_{A_{2} B_{2}}$. Therefore, the interference restriction is relaxed, and the number of broadcast links that can be transmitted concurrently is increased, which is beneficial to improving the performance of information transmission.

In the example of the broadcast link scheduling shown in Figure 9, if the situation shown in Figure 10 exists, node $n_{6}$ is closer to node $n_{10}$; therefore, the GreedyA algorithm analyzes whether node $n_{6}$ will interfere with node $n_{10}$ to receive the signal from node $n_{5}$. As a result, two sending nodes cannot perform data transmission at the same time and need to perform interference avoidance scheduling. However, after combining with the successive interference cancellation technology, the EDTC algorithm analyzes the receiving node $n_{10}$, which can firstly decode the signal of the $n_{6}$ node and then remove the signal. Thus, the signal of node $n_{5}$ is obtained, so the simultaneous data transmission by the two sending nodes will not affect the normal reception of data by the receiving node.

Because in the practical application of the EDTC algorithm, there may be multiple broadcast links. Therefore, it is necessary to analyze the cumulative interference effects of multiple broadcast links; that is, the influence of multiple interference signals needs to be considered in the denominators of formulas (13) and (16).

\subsection{Theoretical Analysis of Algorithm Performance}

\subsubsection{Correctness Analysis}

Theorem 1. The GreedyA algorithm provides a correct broadcast scheduling scheme.

Certification. Consider the entire opportunistic complex networks as a connected network. The GreedyA algorithm first stratifies all nodes, then constructs broadcast tree, and finally uses the method of layer-by-layer scheduling for information transmission. Interference avoidance scheduling allocates the transmission time slice of each sending node. Because the broadcast tree covers all nodes in the entire network, each node has the parent node. That is, broadcast data can be transmitted from the source node to all nodes in the network. Next, it is analyzed whether the forwarding node in the broadcast tree has scheduled its parent node to broadcast data to the node before sending the data. In addition, consider whether the scheduled broadcast links will affect the correct reception of data due to signal interference.

According to the construction rules of the broadcast tree, the parent node of a forwarding node is one level above the node. Because after the ending of scheduling at each layer, the scheduling time slice will be set as the maximum scheduled transmission time slice plus 1 . Therefore, the transmission time slice of the forwarding node must be smaller than the transmission time slice of any node in the previous layer.

The idea of interference avoidance scheduling adopted by the GreedyA algorithm allocates the transmission time slice of the broadcast link. That is, when two broadcast links interfere with each other, the two broadcast links are allocated to different time slices for transmission. We can understand that when two broadcast links interfere with each other, the two broadcast links are allocated to different time slices for transmission. Therefore, the scheduled broadcast links will not affect the correct reception of data due to signal interference.

Theorem 2. The EDTC algorithm provides a correct broadcast scheduling scheme.

Certification. The steps of the EDTC algorithm are similar to the steps of the GreedyA algorithm, and the only difference is the basis for judging whether the link is interfered. According to the basic idea of successive interference cancellation, when the two links have a dependency relationship, the receiving node can correctly decode the required data signal. Therefore, the broadcasting chain scheduled by the EDTC algorithm can also realize the correct reception of data.

\subsubsection{The Analysis of Time Complexity}

Theorem 3. The time complexity of the GreedyA algorithm is $O\left(Y n^{3}\right)$.

Certification. The first step of the EDTC algorithm is to construct a breadth first search tree to stratifies all nodes, and 


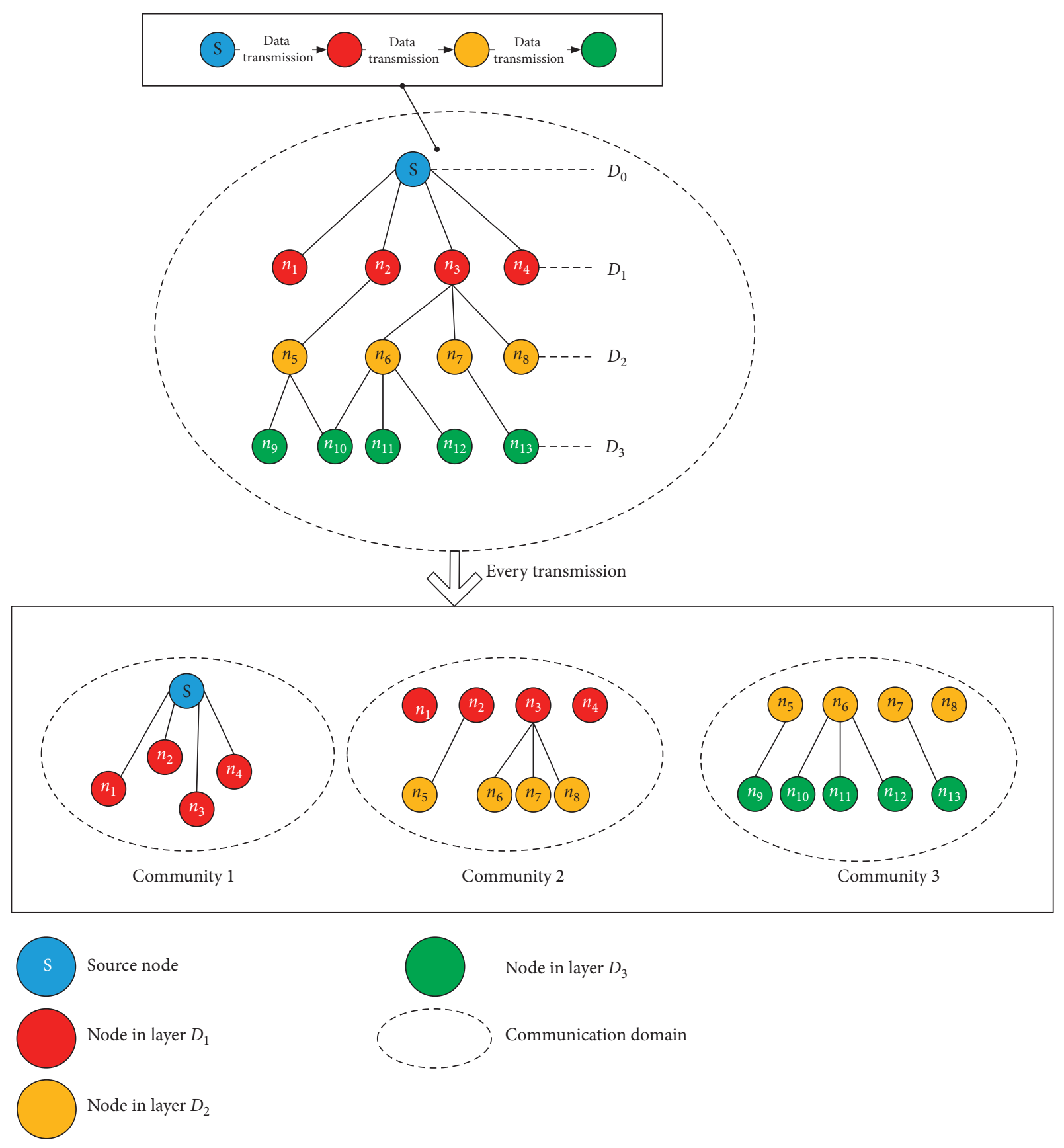

Figure 7: Broadcast tree and three communities.

it needs to traverse each node. So, the time overhead is $O(n)$. The second step is to construct a broadcast tree, which determines the parent-child relationship between nodes. The rule with the largest number of covered nodes needs to analyze the neighbors of each node, so the time overhead is $O\left(n^{2}\right)$; The final step is to broadcast scheduling level by level. When performing broadcast scheduling at each layer, it is necessary to consider whether there is an interference relationship between different broadcast links; therefore, the time overhead of broadcast scheduling for each layer is $O\left(n^{3}\right)$. Because the total number of layers is $Y$, the last step requires $O\left(\mathrm{Yn}^{3}\right)$ time. Therefore, the time overhead of all steps can be summed up to get this theorem.

Theorem 4. The time complexity of the EDTC algorithm is $O\left(Y n^{3}\right)$.

Certification. The steps of EDTC algorithm and GreedyA algorithm are basically the same. The main difference is the basis for judging interference. Therefore, by proof similar to 


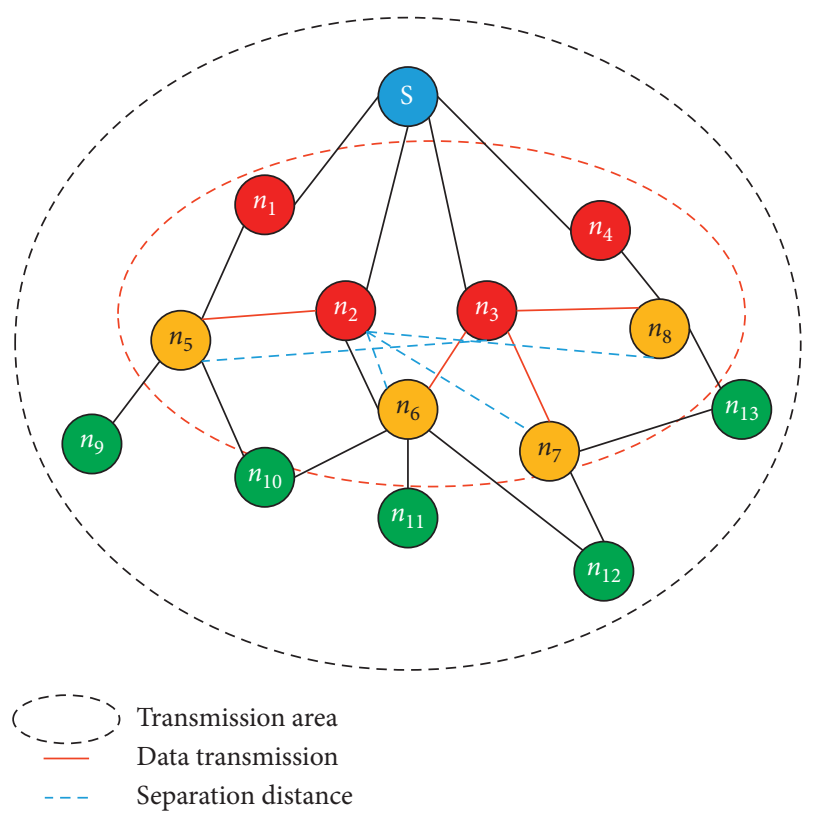

FIgURe 8: Broadcast link scheduling in community 2.

Theorem 4, it can be obtained that the time complexity of the EDTC algorithm is also $O\left(Y n^{3}\right)$.

\subsubsection{The Analysis of Spatial Complexity}

Theorem 5. The spatial complexity of the GreedyA algorithm is $O(n)$.

Certification. Analyze the amount of temporary storage space at each step of the GreedyA algorithm during the working process. The first step is to construct a breadth first search tree and stratify the nodes. The information of the neighbor node needs to be stored during the working process. Because the node has at most $\Phi$ neighbor nodes, the space complexity of this step is $O(\Phi)$. The second step is to construct a broadcast tree. The information of the neighbor nodes is also required to be stored during the working process, so the spatial complexity is $O(\Phi)$. The last step is to perform broadcast scheduling according to the broadcast tree. During the operation, the scheduled broadcast link information needs to be stored. The spatial complexity is $O(n)$. After synthesizing the spatial complexity of all steps, it can be obtained that the spatial complexity of the GreedyA algorithm is $O(n)$.

Theorem 6. The space complexity of the EDTC algorithm is $O(n)$.

Certification. The steps of EDTC algorithm and GreedyA algorithm are basically the same. The main difference is the basis for judging interference. No extra storage space is required in the judgment process. Therefore, this theorem can be established through a certificate similar to Theorem 5 .

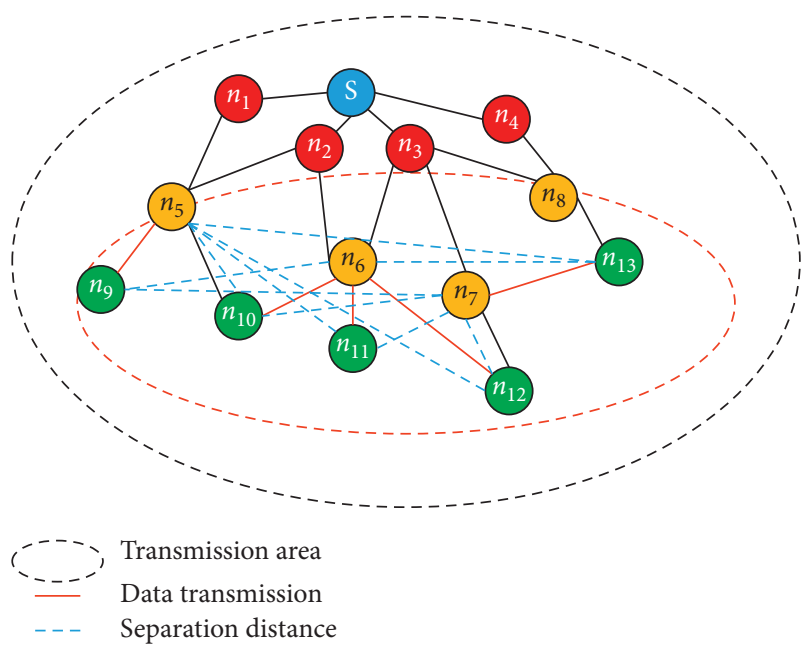

Figure 9: Broadcast link scheduling in community 3.

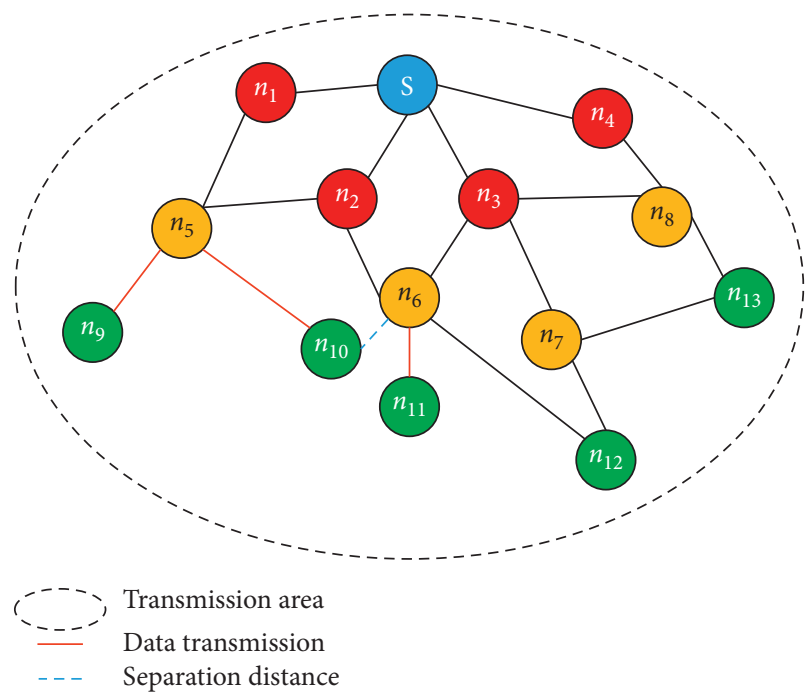

Figure 10: Broadcast link scheduling based on EDTC.

\section{Results and Discussion}

For the evaluation of the experimental performance of the EDTC algorithm, we test it with the opportunistic complex networks environment (ONE). In addition, in order to better judge its performance, EDTC will be compared with four other algorithms: ICMT (information cache management and data transmission algorithm) [1], SECM (status estimation and cache management algorithm) [47], Spray and Wait routing algorithm [36], and GreedyA algorithm. The following is an introduction to the principles of these algorithms:

(1) ICMT: this algorithm is an information cache management and transmission algorithm based on node data information cache. For achieving the purpose of adjusting the cache, the algorithm evaluates the transmission probability between nodes by identifying the neighbor nodes and then adjusts the cache data 
distribution to ensure that nodes with a higher transmission probability have priority to access information. Simultaneously, neighbor nodes share the cache tasks of the nodes and effectively distribute data [1].

(2) SECM: to evaluate the probability of nodes in the project by establishing a method to identify surrounding neighbors, ensuring that nodes with high project probability obtain information first and achieve the purpose of adjusting the cache [47].

(3) Spray and Wait: this algorithm successfully overcomes the shortcomings of epidemic routing and other floodbased schemes, avoiding the performance dilemma inherent in utility-based schemes [36].

(4) In addition, we compare the proposed EDTC algorithm with the GreedyA algorithm. The algorithm we proposed is based on the GreedyA algorithm of the greedy broadcast algorithm and fully utilizes the benefits of successive interference cancellation to schedule the broadcast link in the social network, relaxing the limit of link interference and increasing the number of broadcast links that can be transmitted concurrently. By comparing the simulation experimental data between the two algorithms, we can see more clearly the impact of successive interference cancellation technology on the information transmission in the social network.

In simulation experiments, we mainly evaluate the performance of the algorithm based on the following parameters:

(1) Delivery ratio: this parameter represents the possibility of selecting a relay node during transmission. The delivery ratio is an important indicator in the performance evaluation of opportunistic social networks, which directly reflects the performance of the data distribution mechanism.

(2) Overhead on average: the network overhead of successfully transmitting information between a pair of nodes can be expressed as this parameter, referring to the ratio of the difference between the total number of forwarded message copies in the network and the total number of messages successfully delivered to the destination node.

(3) Energy consumption: this parameter represents the energy consumption during transmission.

(4) End-to-end delay on average: this parameter represents the average delay in transmitting information between two nodes. End-to-end network delay is the time from when a message is generated to when it is successfully delivered to the destination node.

During the simulation experiment, we set its parameters as follows: the maximum transmission distance is $35 \mathrm{~m}$, the signal transmission power is $1300 \mathrm{~W}$, the noise power is $1.5 \mathrm{~W}$, and the signal attenuation index is 2 . Other parameters for environment configuration are shown in Table 1.

In the EDTC algorithm, the size of the specific threshold will directly affect whether a receiving node can decode the
TABLE 1: Experimental parameter settings.

\begin{tabular}{lc}
\hline Parameter & Value \\
\hline Communication area & $3800 \mathrm{~m} \times 3000 \mathrm{~m}$ \\
Total number of nodes & 400 \\
Node movement speed & $0.5 \sim 1.5 \mathrm{~m} / \mathrm{s}$ \\
Node cache storage & $3 \mathrm{MB}$ \\
Simulation time & 5 hours \\
Movement mode & Random site moving model \\
Maximum transmission domain & $15 \mathrm{~m}^{2}$ \\
The interval between each two times & $20-25 \mathrm{~s}$ \\
Initial energy & 100 Joules \\
Each energy depletion & 0.5 Joules \\
\hline
\end{tabular}

obtained signal and remove it from the mixed signal, thereby receiving the required signal. Therefore, different specific thresholds will cause the EDTC algorithm to exhibit different performances during the transmission of information. In order to better explain the relationship between the specific threshold value and transmission performance, this paper uses subjective judgment to set the specific threshold value to $1.5,2.5,3.5,4.5$, and 5.5 for experiments.

First, through Figures 11(a)-11(d), we can find that no matter what the value $\chi_{\text {SIC }}$ is, their delivery ratio, overhead on average, and end-to-end delay on average all tend to stabilize over time. For energy consumption, it gradually increases with time. By comparing the experimental data curves obtained with different thresholds, it can be found that when the value of threshold is too high or too low, the performance of information transmission in social networks is not very ideal. For example, when $\chi S I C=1.5$, the message delivery ratio is only maintained at about 0.56 , and when $\chi S I C=5.5$, the energy consumption is the most. This is because the standard of whether the receiving node can decode the signal is determined by the specific threshold value. When the specific threshold is continuously increased, the conditions for decoding interference signals become more and more severe so that the propagation performance of the EDTC algorithm shows a certain upward trend. However, as the specific threshold continues to increase, the interference signal cannot be effectively decoded, thereby reducing the performance of information transmission. But when the specific threshold value is in the interval $[2.5,3.5]$, a better transmission result can be obtained. In order to better verify the performance of the EDTC algorithm in subsequent experiments, we set the specific threshold to 3.5.

Through the simulation experiments, we found that the relationship between the four parameters to be evaluated and time is shown in Figures 12-15.

Firstly, in Figure 12, we show the relationship between each algorithm's delivery ratio in simulation experiments and simulation experiment time. We can clearly see from the figure that the lowest delivery ratio are Spray and Wait routing algorithms $($ copy $=30)$ and SECM. Their values are $0.31-0.36$ and $0.35-0.39$ separately. We can know that when the Spray and Wait routing algorithm $($ copy $=30)$ and SECM algorithm are used for information transmission, because they use the flooding method to transmit information to the nodes in the community, a large amount of information is 


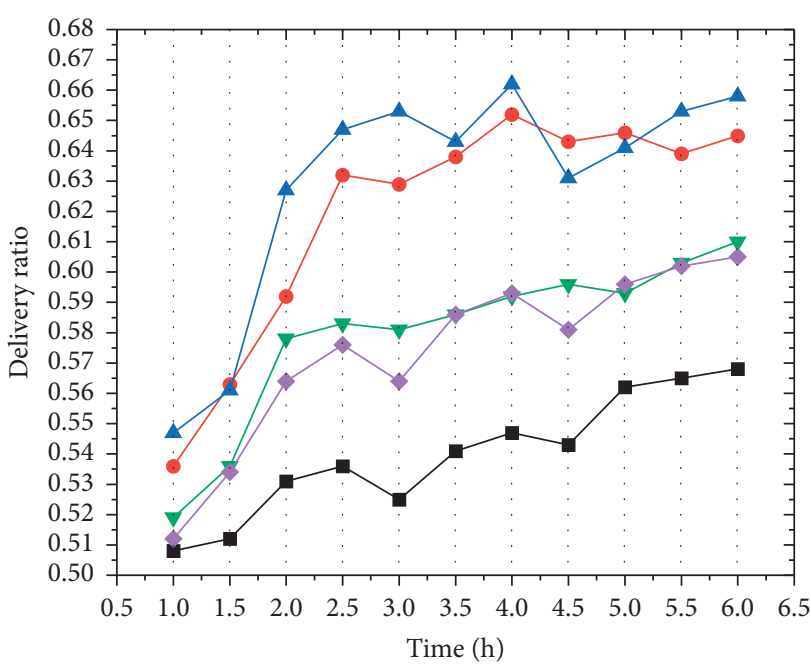

$-\chi \mathrm{S} / \mathrm{C}=1.5$
$-\chi \mathrm{S} / \mathrm{C}=2.5$
$-\chi \mathrm{S} / \mathrm{C}=3.5$

$\nabla \chi \mathrm{S} / \mathrm{C}=4.5$

$\neg \chi \mathrm{S} / \mathrm{C}=5.5$

(a)

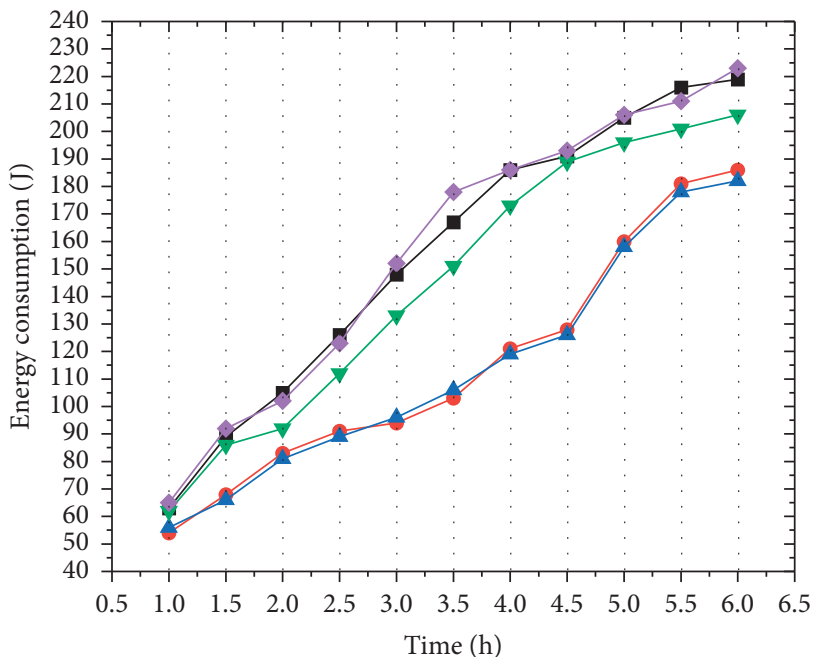

$\rightarrow \chi \mathrm{S} / \mathrm{C}=1.5$
$-\chi \mathrm{S} / \mathrm{C}=2.5$
$-\chi \mathrm{S} / \mathrm{C}=3.5$

$\rightarrow \chi \mathrm{S} / \mathrm{C}=4.5$

$\neg \chi \mathrm{S} / \mathrm{C}=5.5$

(c)

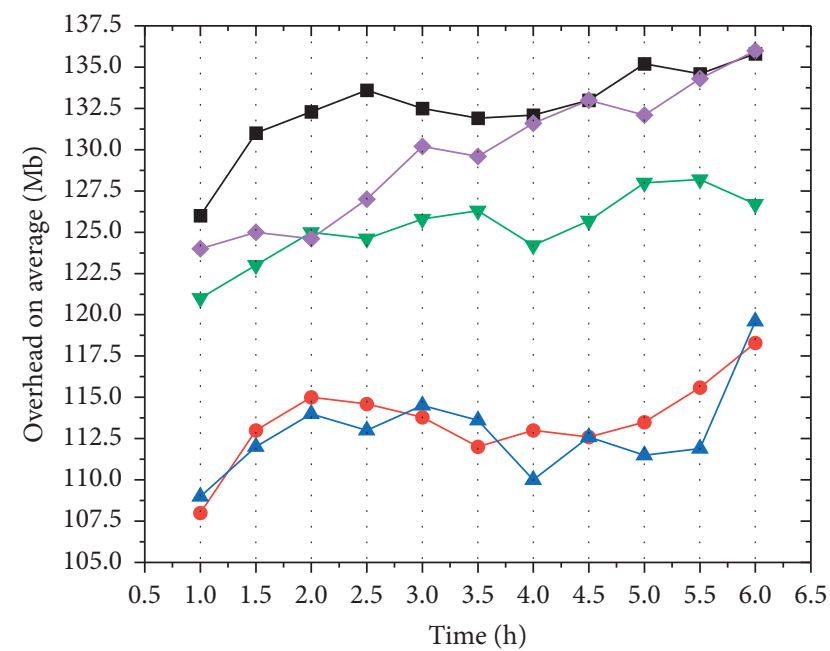

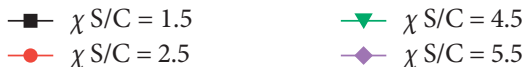

$\leftarrow \chi \mathrm{S} / \mathrm{C}=3.5$

(b)

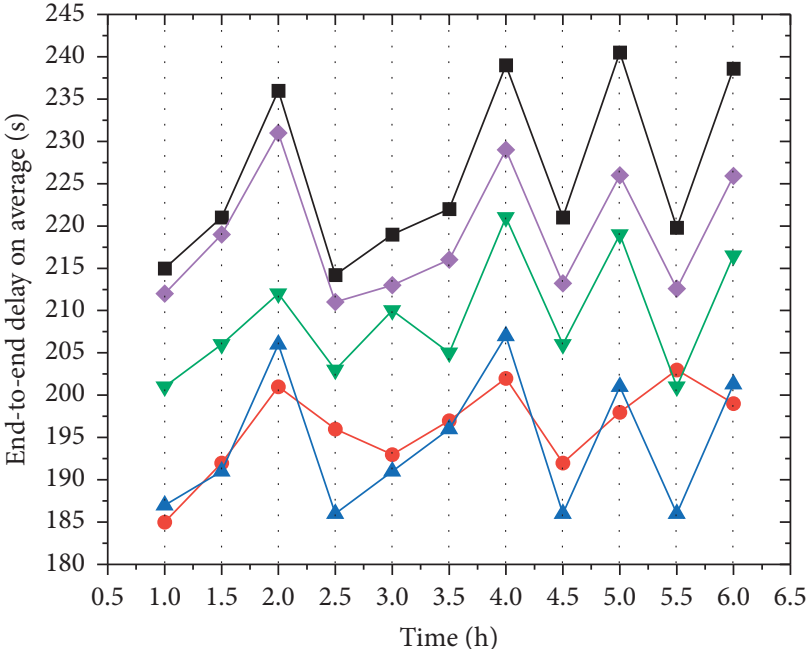

$\rightarrow \chi \mathrm{S} / \mathrm{C}=1.5$
$\rightarrow \chi \mathrm{S} / \mathrm{C}=2.5$
$-\chi \mathrm{S} / \mathrm{C}=3.5$

$\checkmark \chi \chi \mathrm{S} / \mathrm{C}=4.5$

$\diamond \chi \mathrm{S} / \mathrm{C}=5.5$

(d)

FIGURE 11: The relationship between each parameter value and time under different specific thresholds $\chi_{\text {SIC }}$.

lost in the process. Especially for the Spray and Wait routing algorithms, we can see that when the number of copies is 30 , its delivery ratio is significantly lower than when the number of copies is 15 . The delivery ratio of the Spray and Wait routing algorithms $($ copy $=15)$ is $0.41-0.47$. Therefore, the excessive copying of data is an important reason for the reduction of the delivery ratio of the Spray and Wait routing algorithm. However, in the ICMT algorithm, the transmission of all its packets depends on the cooperation of the cache, which effectively uses the cache space. This achieves the purpose of increasing the delivery ratio. It can be found from Figure 12 that the delivery ratio of the experimental data using the ICMT algorithm is $0.53-0.59$, which is $147 \%$ more than SECM. As for the EDTC algorithm proposed in this paper, because when information is transmitted, the nodes in the same community are used to construct a broadcast tree, and the appropriate next-hop node is selected for hierarchical propagation through the coverage. In this way, the reliability and value of the nodes are comprehensively considered, and the node with the highest comprehensive utility value is accurately found, which avoids signal interference when data are propagated in parallel and also guarantee the probability of the message reaching the destination node. Because of this, it has greatly improved the delivery ratio in social networks. It can be seen that its delivery ratio reaches 0.66 , which is the highest among these 


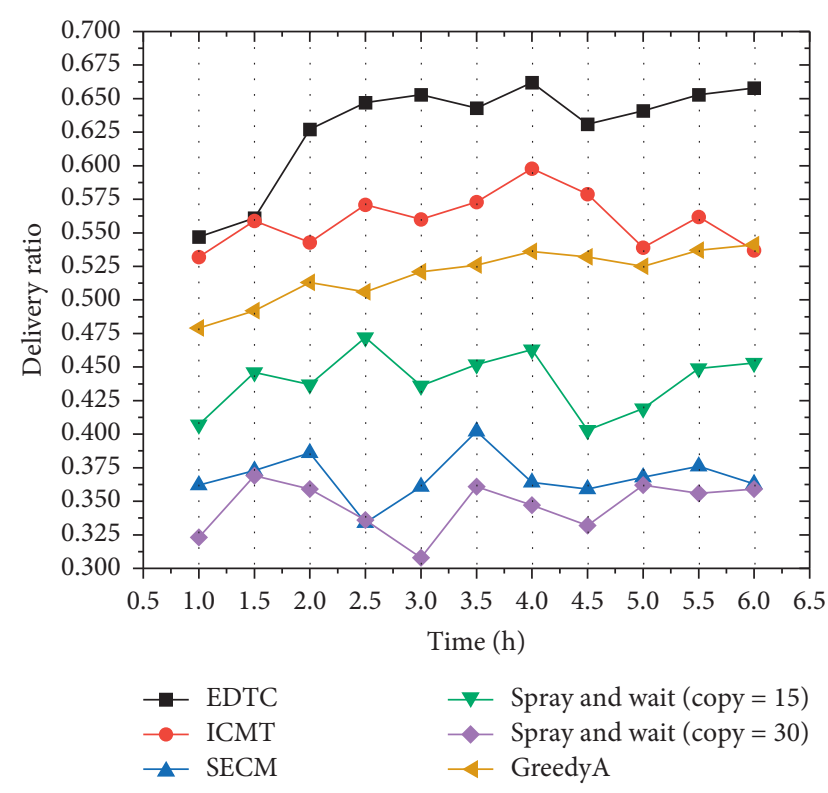

FIgURE 12: The relationship between delivery ratio and time.

algorithms. For the GreedyA algorithm, because it is limited by the number of broadcast links that can be transmitted concurrently, the delivery ratio is lower than the EDTC algorithm. However, because it uses the layer-by-layer scheduling and interference avoidance scheduling methods, it allocates the transmission time slices of the broadcast link, which effectively solves the signal interference problem, so its delivery ratio is stronger than the Spray and Wait and SECM algorithms, which is in a relatively high level.

From Figure 13, we can get the relationship between routing overhead and time. In the transmission algorithms of social networks, if there is a lack of a cache management method to maintain information transmission when a node meets its neighbors, a large amount of redundant data will be received in the cache, thus increasing the overhead. Therefore, in the simulation experiments, the overhead on average obtained by applying the SECM algorithm and the Spray and Wait algorithms is high. For the ICMT algorithm, the overall mean overhead is low and stable, but it increased in the early stage, reaching its peak in 2 hours, and then began to decline and stabilize. Because when using the EDTC algorithm to transmit information, through the constructed broadcast tree, messages can always be delivered to the correct node, thereby avoiding forwarding to unnecessary nodes and reducing the number of copies of forwarded messages in the network. Those improve network congestion and effectively reduce network overhead. Like the EDTC algorithm, the overhead on average of the GreedyA algorithm is also maintained at an ideal level.

Figure 14 shows the connection between energy consumption and stimulation time. In simulation experiments, all energy consumption increases with time. Among them, because the Spray and Wait routing algorithms need to transmit information through spray, its energy consumption is the largest. It can be seen from Figure 14 that, at the 6 th hour, its energy consumption reached 540. For the ICMT

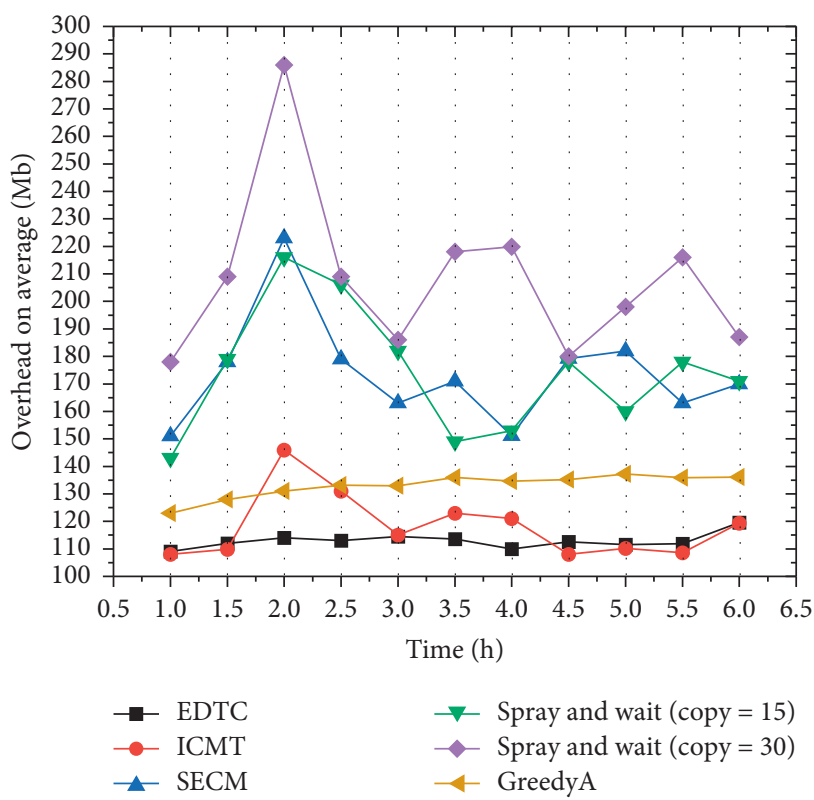

FIGURE 13: Relationship between routing overhead and time.

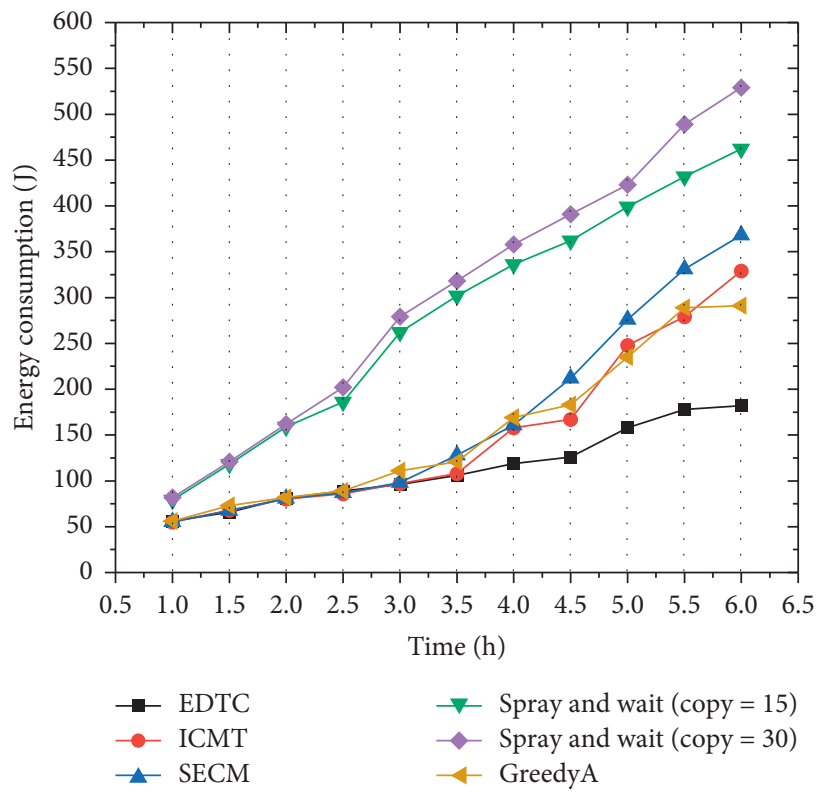

FIgURE 14: Relationship between energy consumption and time.

algorithm, because it can exchange valid data through cooperative nodes, it retains more energy to continue transmission, so its energy consumption data can maintain a low level. The energy consumption data obtained by applying the SECM algorithm is similar to the ICMT algorithm. For the EDTC algorithm, it is different from flooding to achieve the highest delivery rate without any cost for data transmission, resulting in a large amount of energy consumption during data transmission. The EDTC and GreedyA algorithm spreads information transmission by selecting the most appropriate nodes in a hierarchical manner, thereby ensuring the delivery rate and keeping consumption low. 


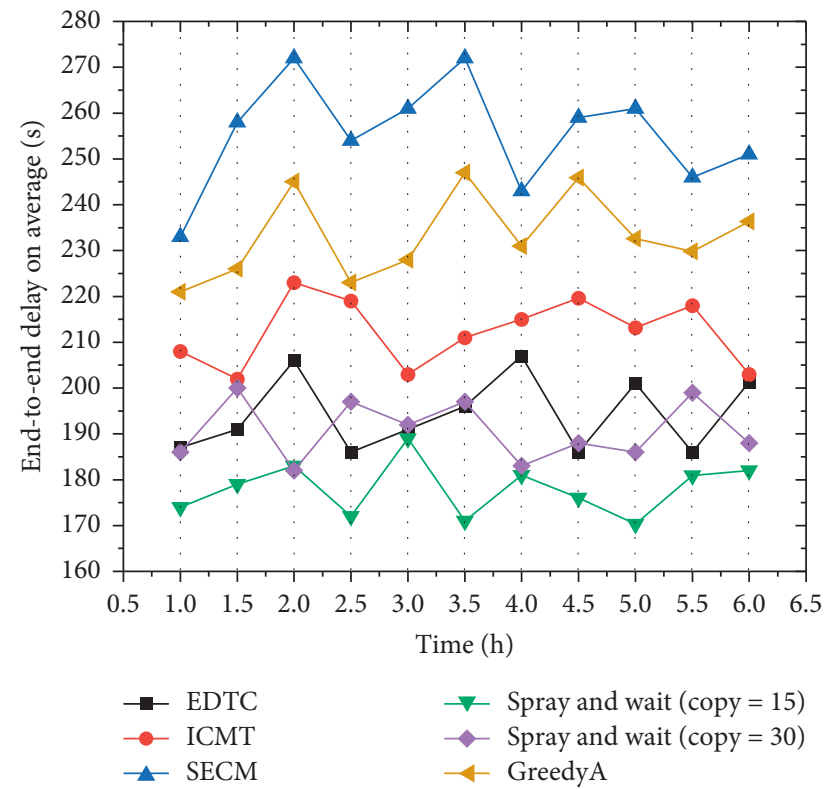

FIGURE 15: Relationship between end-to-end delay on average and time.

In Figure 15, we show the relationship between mean transmission delay and time. It can be seen from Figure 15 that the transmission delay in the social network using the SECM algorithm is very high mainly because the spray step is carried by nodes. The ICMT algorithm uses a cooperative mechanism to achieve reasonable utilization of node cache space. This reduces the propagation delay, but the effect is not very obvious. It is not difficult to find that the best algorithm to reduce the transmission delay among these algorithms is Spray and Wait routing algorithms, which is mainly because neighbors and cooperative nodes are used when data are transferred between nodes, and a large number of shared caches can be used in transmission. According to the broadcast characteristics of wireless signals, nodes only need to broadcast once to transmit data to nodes within coverage. For our proposed EDTC algorithm, it is a broadcast tree constructed according to the rule that the number of covering nodes has the highest priority as the parent node. In addition, the signal interference is avoided through successive interference cancellation technology so as to increase the number of simultaneous transmissions. As shown in Figure 15, its mean transmission delay is maintained at a very low value. Compared with the EDTC algorithm, the GreedyA algorithm has a lower number of broadcast links that can be transmitted concurrently, so its broadcast delay is higher than the EDTC algorithm.

In summary, in the four aspects of delivery ratio, overhead on average, energy consumption, and end-to-end delay on average, we can conclude that the performance of EDTC is better than other four algorithms. However, for the end-to-end delay on average, the performance of EDTC is worse than Spray and Wait routing algorithms.

In social networks, node cache has a great impact on the transmission efficiency of the algorithm. Therefore, we continue to do simulation experiments to test the influence

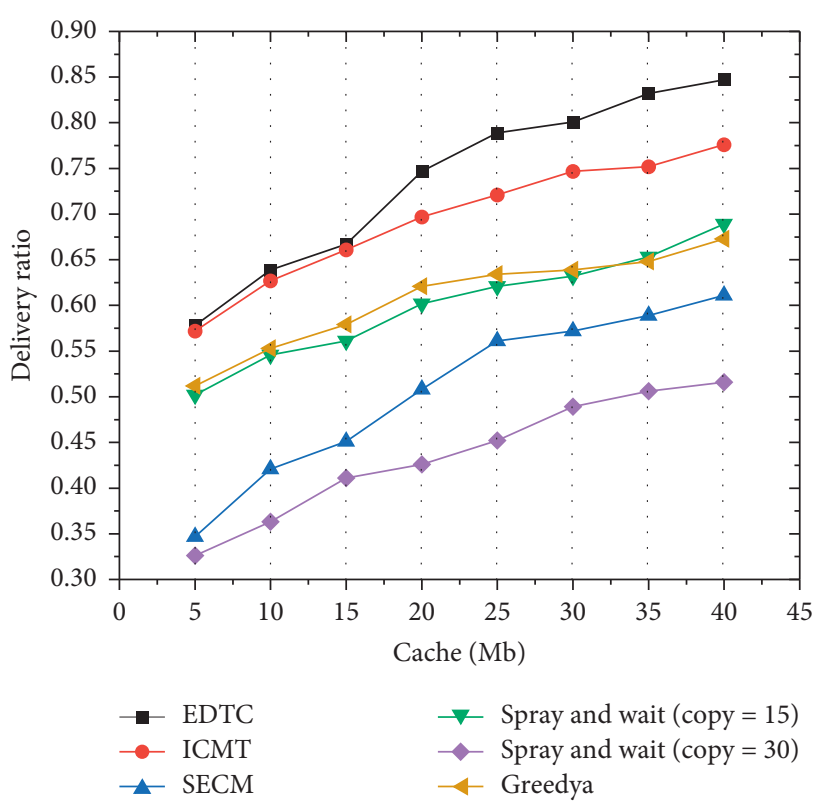

FIgURe 16: Relationship between delivery ratio and cache.

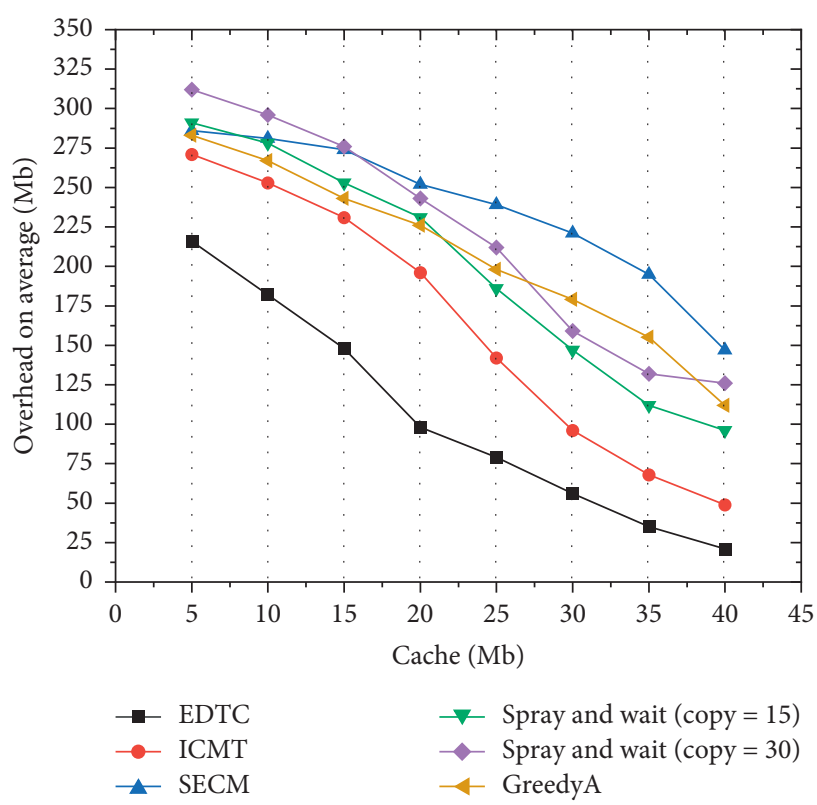

FIGURE 17: Relationship between overhead on average and cache.

of cache on these four parameters. The experimental data that we obtained are shown in Figures 16-19.

In Figure 16, we show the relationship between delivery ratio and cache. When the buffer is small, the network cannot meet the message cache requirements due to the large number of message copies, and the old messages will be quickly squeezed out by the new ones, causing a large number of packets to be dropped. Therefore, the delivery ratio of the five algorithms is not high when the cache is low. But with the increase in the buffer capacity, the transmission rate has increased to varying degrees. Among them, because the Spray and Wait routing algorithms $($ copy $=30)$ use the method of 
flooding to spread information and require high cache size, its delivery ratio is the lowest. However, for the EDTC and GreedyA algorithm, nodes only need to broadcast once to transmit data to nodes within coverage. Because the GreedyA algorithm is limited by the number of concurrent broadcast links, the delivery ratio is slightly lower. It can be seen from the experimental results that EDTC has the highest delivery ratio. As for the application of ICMT and Spray and Wait routing algorithms $($ copy $=15)$ in the process of transmitting information on opportunistic complex networks, with the increase in the node cache, the transportation conditions improved, and the delivery ratio increased by more than $50 \%$.

The association between rooting overhead and cache is shown in Figure 17. In general, as the cache increases, the packet loss of nodes in the network becomes smaller, more messages can be successfully transmitted, and the overhead is getting lower and lower. As shown in Figure 17, the overhead of SECM algorithm is the largest because many redundant data are injected by nodes. As the node cache increases, the overhead on average applying the Spray and Wait routing algorithms (copy $=30)$ is reduced from 310 to 136 . Similarly, the overhead of Spray and Wait routing algorithms (copy $=15$ ) is reduced from 281 to 98 . But their percentage of decline is much lower than the EDTC and ICMT algorithms. It can be found that, by increasing the node cache, the purpose of decreasing the routing overhead of the nodes in the community can be achieved. As the cache increases, the overhead on average of GreedyA algorithm also decreases from 280 to 117.

The relationship between energy consumption and cache is shown in Figure 18. The experimental results show that, with the increase in node cache, the energy consumption of EDTC algorithm in opportunistic complex networks can be maintained at 48. GreedyA also has a good performance, and energy consumption is stable at 76 . The energy consumption of the other three algorithms has increased significantly. Because the Spray and Wait routing algorithms use the "Spray" method and all neighbors receive the data packets, its energy consumption is the largest. In two experiments using the Spray and Wait routing algorithms, the Spray and Wait routing algorithms $($ copy $=30)$ with a larger copy data consumes more energy. For the ICMT algorithm, the effect buffer management method can cut down energy consumption. So its energy consumption is less than the Spray and Wait routing algorithms.

As shown in Figure 19, the relationship between the average delivery delay and the cache can be obtained. Figure 19 shows that the mean delay decreases as the node cache increases. For the SECM algorithm, the average delay is generally high, which is mainly because many probability calculation tasks are carried by nodes. For the ICMT algorithm, as the node cache increases, the average delay decreases from 178 to 56 . In the EDTC algorithm, the mean delay is almost stable at 41 . This shows that the size of the cache has a small impact on the average delay of the EDTC algorithm. In addition, the delay generated by the greedy algorithm is higher, which shows that multiple concurrent links generated by successive interference cancellation techniques can reduce the delay of information transmission in social networks. For the Spray and Wait routing algorithms, although the average delay decreases with the

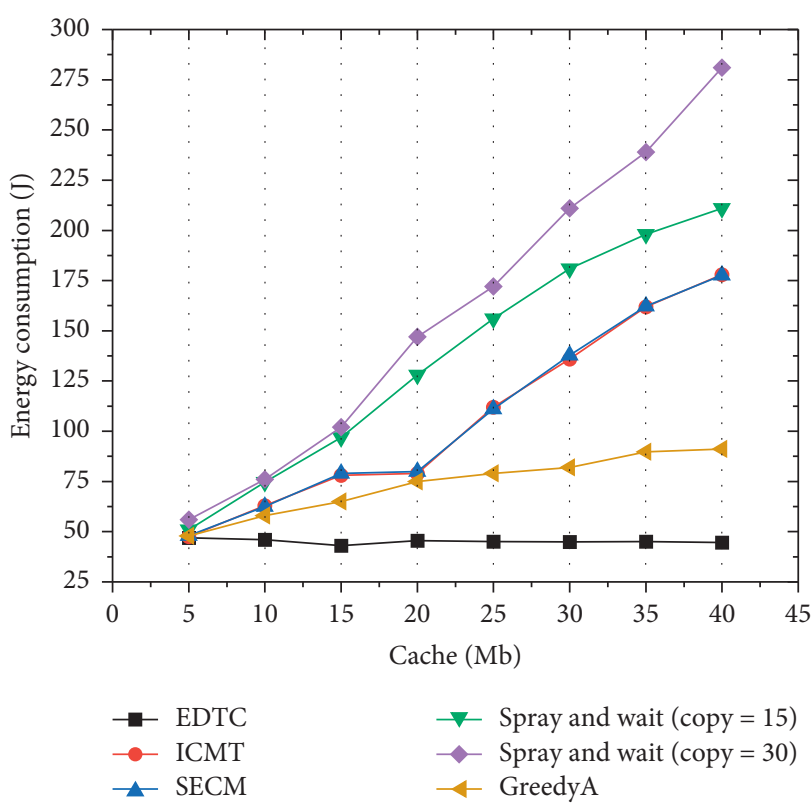

FIGURE 18: Relationship between energy consumption and cache.

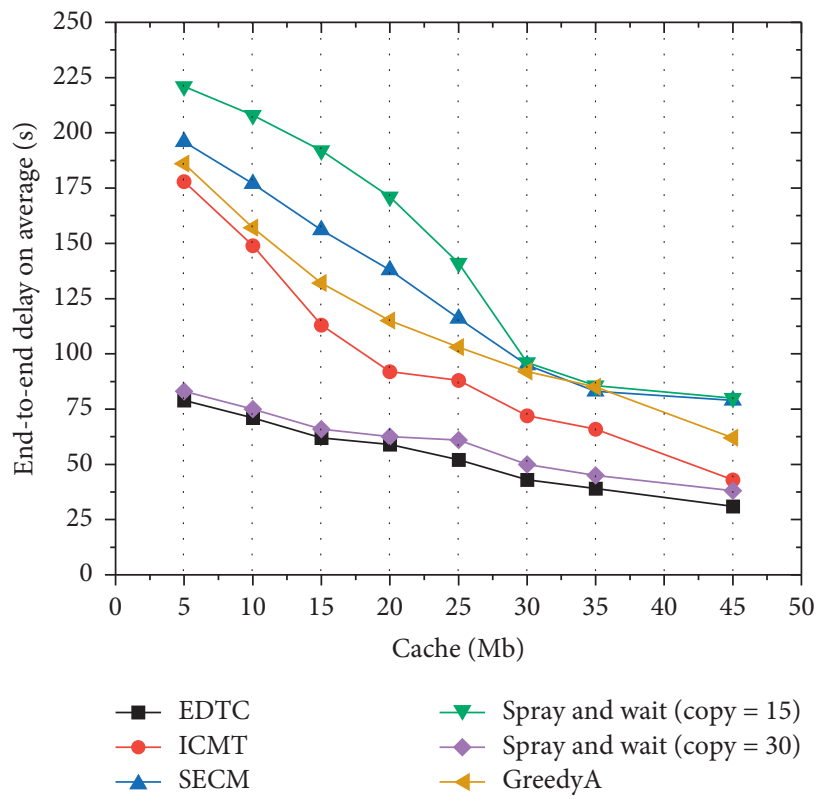

FIgURE 19: Relationship between end-to-end delay on average and cache.

increase in the node cache. However, in the case of copy $=30$, the mean delay is significantly lower than the copy $=15$.

In actual social networks, the choice of information transfer methods also has a great impact on the performance of the algorithm. So in order to test our proposed EDTC algorithm, in the following simulation experiments, we choose three different models to evaluate the performance of the EDTC algorithm. These three models are SPMBM (shortest path map-based movement), random way point (RWP), and random walk (RM) models [48].

Figure 20 shows the change in deliver ratio in the EDTC algorithm of different mobile models. It can be found from 


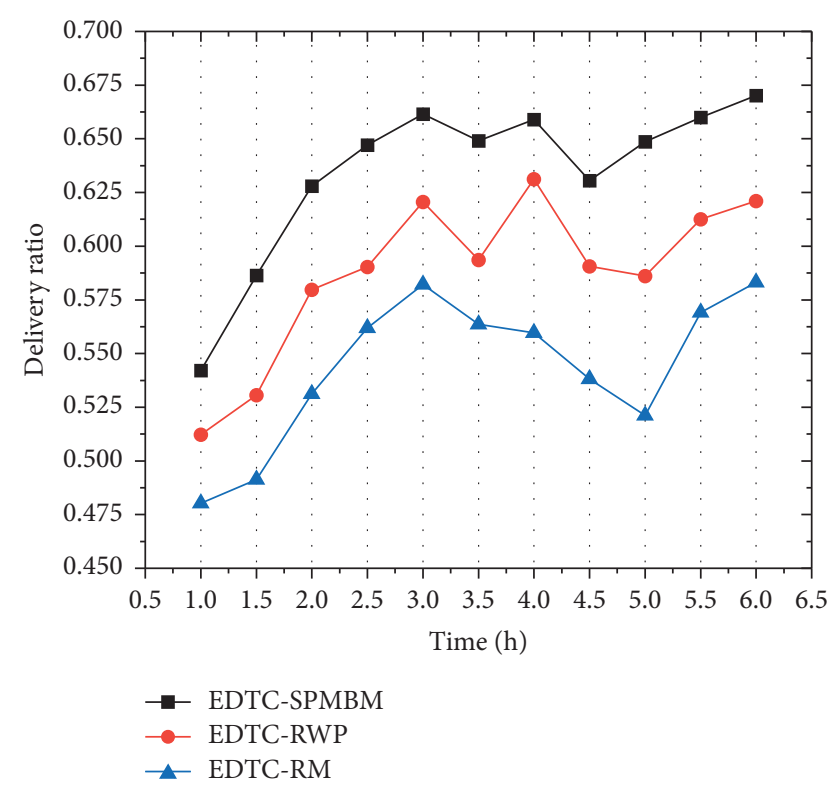

FIgUre 20: Relationship between delivery ratio and time in three mobile models.

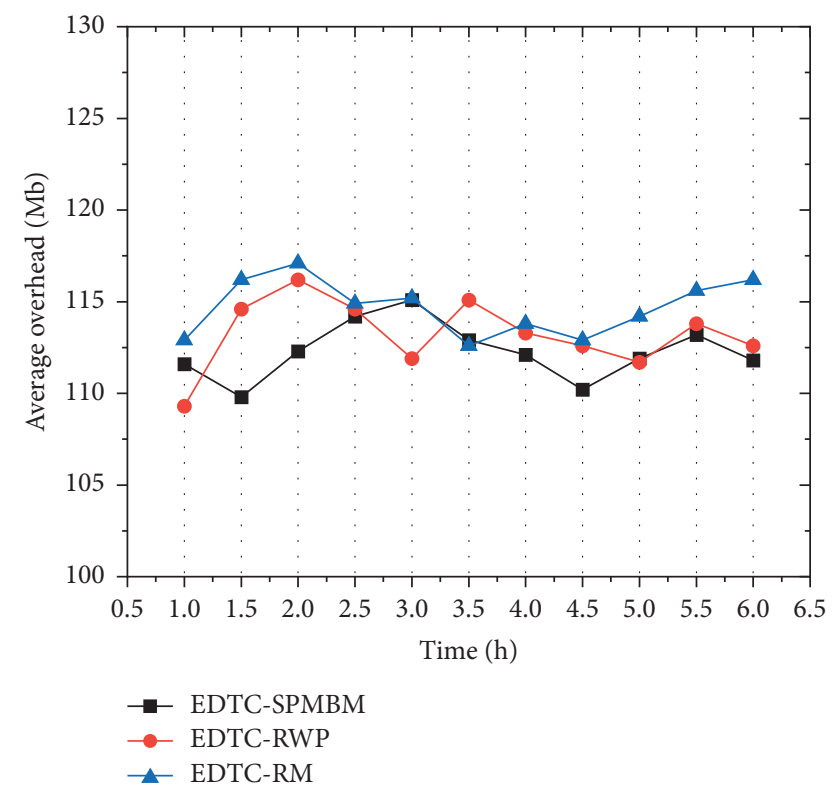

FIGURE 21: Relationship between average delay and time in three mobile models.

Figure 20 that the delivery ratio obtained under the SPMBM model is the highest, finally reaching 0.679 . For the experiment using the RM model, the delivery ratio reached the peak 0.583 in the 6th hour. However, in the case of applying the RWP model, the peak 0.626 reached at the 4 th hour. In general, the delivery ratio of the EDTC algorithm in the SPMBM model is higher than in RM and RWP models. Moreover, the RWP model is better than the RM model.

We can get the transformation of EDTC algorithm's routing overhead in different models from Figure 21. In general, in the process of applying EDTC algorithm for information transmission under these three models, the overhead

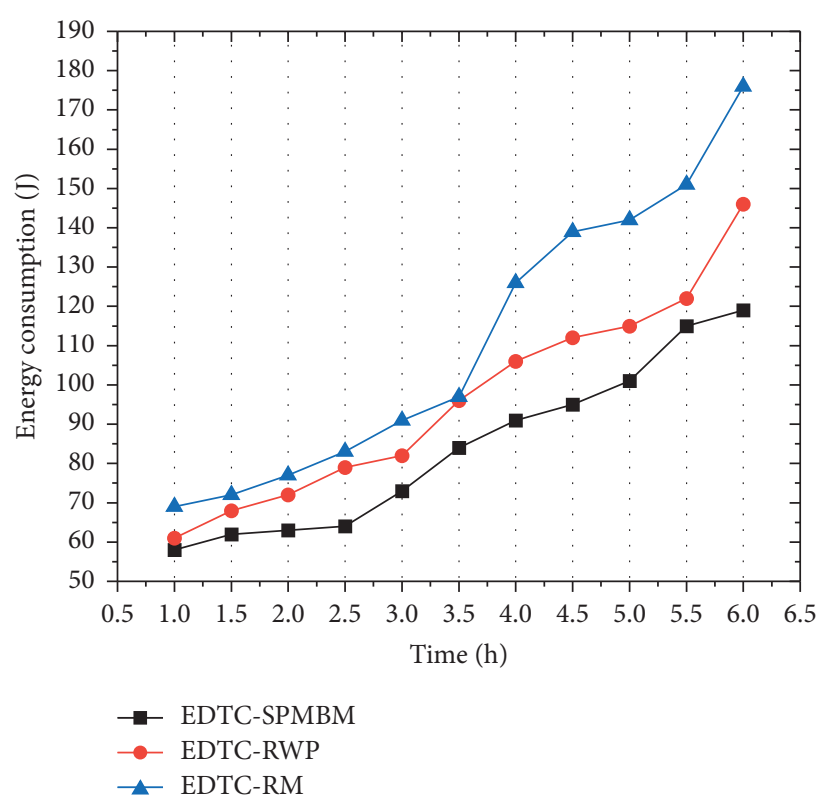

FIGURE 22: Relationship between energy consumption and time in three mobile models.

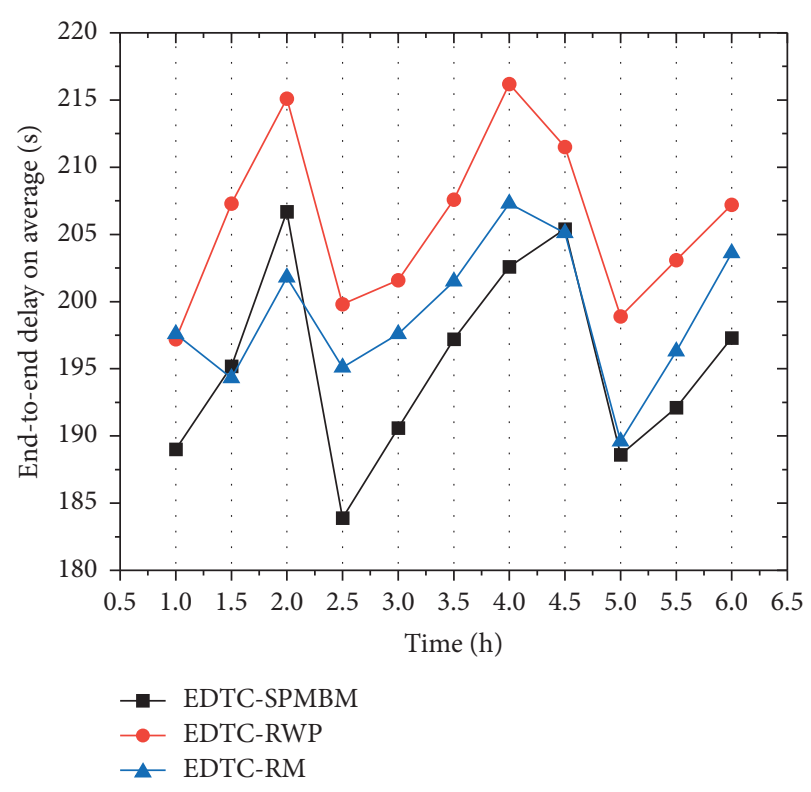

FIgURE 23: Relationship between end-to-end delay on average and time in three mobile models.

change with time is relatively small, and it remains in the range of 108 117. This experiment shows that the choice of different models has little effect on the delay caused by the EDTC algorithm for information transmission.

Figure 22 shows the difference in energy consumption over time for different models. In general, the energy consumption in all three models increases with time. The difference in energy consumption between the three models is small. The results show that the EDTC algorithm has stable node information transmission performance and does not consume a lot of energy when the model changes. 
We can get the information of EDTC algorithm's average delay in different models from Figure 23. The mean delay obtained by transmission under the three models is almost floating between 183 and 216, which shows that the EDTC algorithm can effectively transfer information.

\section{Conclusion}

In this paper, we propose an opportunistic complex networks data transmission and control based on successive interference cancellation techniques. This algorithm performs broadcast link scheduling through layer-by-layer scheduling and interference avoidance scheduling. The link scheduling strategy is designed in conjunction with successive interference cancellation techniques to increase the number of broadcast links that can be transmitted simultaneously. This effectively solves the problem of signal interference during data transmission in an opportunity-complex network. And in the experimental stage, we compared the proposed algorithm with other classic algorithms of opportunistic complex networks. Experimental results show that the algorithm has good transmission ability. And relative to the GreedyA algorithm, because it increases the number of broadcast links that can be transmitted concurrently, it achieves better transmission performance. Subsequently, with the purpose of testing the impact of different mobile models on the EDTC algorithm, we, respectively, tested the algorithm based on the SPMBM, RWP, and RM models. Experimental results show that the algorithm has excellent performance in each model. Applying this algorithm to information transmission in opportunistic complex networks can reduce node energy consumption and propagation delay and greatly improve data transmission efficiency. In the future work, this method can adopt to big data environment to solve the problem in transmission.

\section{Data Availability}

Data used to support the findings of this study are currently under embargo, while the research findings are commercialized. Requests for data, 12 months after publication of this article, will be considered by the corresponding author.

\section{Conflicts of Interest}

The authors declare that they have no conflicts of interest.

\section{Acknowledgments}

This study was supported by the National Natural Science Foundation of China (61672540) and Hunan Provincial Natural Science Foundation of China (2018JJ3299 and 2018JJ3682).

\section{References}

[1] J. Wu, Z. Chen, and M. Zhao, "Information cache management and data transmission algorithm in opportunistic social networks," Wireless Networks, vol. 25, no. 6, pp. 2977-2988, 2019.
[2] Y. Wang and J. Wu, "Social-tie-based information dissemination in mobile opportunistic social networks," in Proceedings of the 2013 IEEE 14th International Symposium on "A World of Wireless, Mobile and Multimedia Networks", Madrid, Spain, June 2013.

[3] C. Jiang, Y. Chen, and K. J. R. Liu, "Evolutionary dynamics of information diffusion over social networks," IEEE Transactions on Signal Processing, vol. 62, no. 17, pp. 4573-4586, 2014.

[4] J. Luo, J. Wu, and Y. Wu, "Advanced data delivery strategy based on multiperceived community with IoT in social complex networks," Complexity, vol. 2020, Article ID 3576542, 15 pages, 2020.

[5] Q. Xu, Z. Su, K. Zhang, P. Ren, and X. S. Shen, "Epidemic information dissemination in mobile social networks with opportunistic links," IEEE Transactions on Emerging Topics in Computing, vol. 3, no. 3, pp. 399-409, 2015.

[6] Z. Li, F. Xiong, X. Wang, Z. Guan, and H. Chen, "Mining heterogeneous influence and indirect trust for recommendation," Access IEEE, vol. 8, pp. 21282-21290, 2020.

[7] J. Wu, Z. Chen, and M. Zhao, "Effective information transmission based on socialization nodes in opportunistic networks," Computer networks, vol. 129, pp. 297-305, 2017.

[8] K. Zhu, W. Li, X. Fu, and L. Zhang, "Data routing strategies in opportunistic mobile social networks: taxonomy and open challenges," Computer Networks, vol. 93, pp. 183-198, 2015.

[9] A. Socievole, E. Yoneki, F. De Rango, and J. Crowcroft, "MLSOR: message routing using multi-layer social networks in opportunistic communications," Computer Networks, vol. 81, pp. 201-219, 2015.

[10] J. Wu, Z. Chen, and M. Zhao, "Community recombination and duplication node traverse algorithm in opportunistic social networks," Peer-To-Peer Networking and Applications, vol. 13, no. 3, pp. 940-947, 2020.

[11] C. Jiang, Y. Chen, and K. J. R. Liu, "Graphical evolutionary game for information diffusion over social networks," IEEE Journal of Selected Topics in Signal Processing, vol. 8, no. 4, pp. 524-536, 2014.

[12] Z. Li, F. Xiong, X. Wang, H. Chen, and X. Xiong, "Topological influence-aware recommendation on social networks," Complexity, vol. 2019, Article ID 6325654, 12 pages, 2019.

[13] M. R. Schurgot, C. Comaniciu, and K. Jaffres-Runser, "Beyond traditional DTN routing: social networks for opportunistic communication," IEEE Communications Magazine, vol. 50, no. 7, pp. 155-162, 2012.

[14] D. Zhang, Z. Wang, B. Guo, X. Zhou, and V. Raychoudhury, "A dynamic community creation mechanism in opportunistic mobile social networks," in Proceedings of the 2011 IEEE Third International Conference on Privacy, Security, Risk and Trust and 2011 IEEE Third International Conference on Social Computing, October 2011.

[15] J. Wu, Z. Chen, and M. Zhao, "An efficient data packet iteration and transmission algorithm in opportunistic social networks," Journal of Ambient Intelligence and Humanized Computing, pp. 1-17, 2019.

[16] R. Gandhi, A. Mishra, and S. Parthasarathy, "Minimizing broadcast latency and redundancy in ad hoc networks," IEEE/ACM Transactions On Networking, vol. 16, no. 4, pp. 840-851, 2008.

[17] S. C.-H. Huang, H.-C. Wu, and S. S. Iyengar, "Multisource broadcast in wireless networks," IEEE Transactions on Parallel and Distributed Systems, vol. 23, no. 10, pp. 1908-1914, 2012.

[18] C. S. Vaze and M. K. Varanasi., "The degree-of-freedom regions of MIMO broadcast, interference, and cognitive radio channels with no CSIT," IEEE Transactions on Information Theory, vol. 58, no. 8, pp. 5354-5374, 2012. 
[19] D. Zhao, K.-W. Chin, and R. Raad, "Approximation algorithms for broadcasting in duty cycled wireless sensor networks," Wireless Networks, vol. 20, no. 8, pp. 2219-2236, 2014.

[20] F. Xiong, Y. Liu, L. Wang, and X. Wang, "Analysis and application of opinion model with multiple topic interactions," Chaos: An Interdisciplinary Journal of Nonlinear Science, vol. 27, no. 8, Article ID 083113, 2017.

[21] F. Xiong and Z.-Y. Li, "Effective methods of restraining diffusion in terms of epidemic dynamics," Scientific Reports, vol. 7, no. 1, pp. 1-14, 2017.

[22] F. Xiong, Y. Liu, and J. Cheng, "Modeling and predicting opinion formation with trust propagation in online social networks," Communications in Nonlinear Science and Numerical Simulation, vol. 44, pp. 513-524, 2017.

[23] J. Wu, G. Yu, and P. Guan, "Interest characteristic probability predicted method in social opportunistic networks," IEEE ACCESS, vol. 7, no. 1, pp. 59002-59012, 2019.

[24] D. Yu, Q.-S. Hua, Y. Wang, J. Yu, and F. C. M. Lau, "Efficient distributed multiple-message broadcasting in unstructured wireless networks," in Proceedings of the 2013 Proceedings IEEE INFOCOM, April 2013.

[25] J. Wu, X. Tian, and Y. Tan, "Hospital evaluation mechanism based on mobile health for IoT system in social networks," Computers in Biology and Medicine, vol. 109, pp. 138-147, 2019.

[26] S. Pan, R. Hu, S.-F. Fung, G. Long, J. Jiang, and C. Zhang, "Learning graph embedding with adversarial training methods," IEEE Transactions on Cybernetics, vol. 50, no. 6, pp. 2475-2487, 2019.

[27] S. Pan, R. Hu, G. Long, J. Jiang, L. Yao, and C. Zhang, "Adversarially regularized graph autoencoder for graph embedding," 2018, http://arxiv.org/abs/1802.04407.

[28] L. Wang, Z. Yu, F. Xiong, D. Yang, S. Pan, and Z. Yan, "Influence spread in geo-social networks: a multiobjective optimization perspective," IEEE Transactions on Cybernetics, pp. 1-13, 2019.

[29] L. Wang, Z. Yu, D. Yang, T. Ku, B. Guo, and H. Ma, "Collaborative mobile crowdsensing in opportunistic D2D networks," ACM Transactions on Sensor Networks, vol. 15, no. 3, pp. 1-30, 2019.

[30] H. Liang and T. Baldwin, "A probabilistic rating auto-encoder for personalized recommender systems," in Proceedings of the 24th ACM International on Conference on Information and Knowledge Management, Melbourne, Australia, 2015.

[31] H. Chen, X. Wang, S. Pan, and F. Xiong, "Identify topic relations in scientific literature using topic modeling," IEEE Transactions on Engineering Management, pp. 1-13, 2019.

[32] A. Vahdat and D. Becker, "Epidemic routing for partially connected Ad Hoc networks," Technical Report CS-2000-06, Duke University, Durham, NC, USA, 2000.

[33] H. Lenando and M. Alrfaay, "EpSoc: social-based epidemicbased routing protocol in opportunistic mobile social network," Mobile Information Systems, vol. 2018, Article ID 6462826, 8 pages, 2018.

[34] P. Mundur, M. Seligman, and G. Lee, "Epidemic routing with immunity in delay tolerant networks," in Proceedings of the MILCOM 2008-2008 IEEE Military Communications Conference, November 2008.

[35] F. D. Rango, S. Amelio, and P. Fazio, "Enhancements of epidemic routing in delay tolerant networks from an energy perspective," in Proceedings of the International Wireless Communications \& Mobile Computing Conference, Sardinia, Italy, July 2013.
[36] T. Spyropoulos, K. Psounis, and C. S. Raghavendra, "Spray and wait: an efficient routing scheme for intermittently connected mobile networks," in Proceedings of the 2005 ACM SIGCOMM Workshop on Delay-Tolerant Networking, Philadelphia, PA, USA, 2005.

[37] J. Xue, X. Fan, Y. Cao, J. Fang, and J. Li, "Spray and wait routing based on average delivery probability in delay tolerant network," in Proceedings of the 2009 International Conference on Networks Security, Wireless Communications and Trusted Computing, vol. 2, April 2009.

[38] W. Huang, S. Zhang, and W. Zhou, "Spray and wait routing based on position prediction in Opportunistic Complex Networks," in Proceedings of the 2011 3rd International Conference on Computer Research and Development, vol. 2, March 2011.

[39] S. Jain, M. Chawla, V. N. G. J. Soares, and J. J. Rodrigues, "Enhanced fuzzy logic-based spray and wait routing protocol for delay tolerant networks," International Journal of Communication Systems, vol. 29, no. 12, pp. 1820-1843, 2016.

[40] S. K. Dhurandher, D. K. Sharma, I. Woungang, and S. Bhati, "HBPR: history based prediction for routing in infrastructureless Opportunistic Complex Networks," in Proceedings of the 2013 IEEE 27th International Conference on Advanced Information Networking and Applications (AINA), March 2013.

[41] C. Yu, Z. Tu, D. Yao, F. Lu, and H. Jin, "Probabilistic routing algorithm based on contact duration and message redundancy in delay tolerant network," International Journal of Communication Systems, vol. 29, no. 16, pp. 2416-2426, 2016.

[42] G. Yu, Z. Chen, and J. Wu, "Quantitative social relations based on trust routing algorithm in opportunistic social network," EURASIP Journal on Wireless Communications and Networking, vol. 2019, no. 1, 2019.

[43] F. Xu, Q. Xu, Z. Xiong et al., "Intelligent distributed routing scheme based on social similarity for mobile social networks," Future Generation Computer Systems, vol. 96, pp. 472-480, 2019.

[44] X. Tian, J. Yu, L. Ma, G. Li, and X. Cheng, "Distributed deterministic broadcasting algorithms under the sinr model," in Proceedings of the IEEE INFOCOM 2016-The 35th Annual IEEE International Conference on Computer Communications, April 2016.

[45] N. I. Miridakis and D. D. Vergados, "A survey on the successive interference cancellation performance for single-antenna and multiple-antenna OFDM systems," IEEE Communications Surveys \& Tutorials, vol. 15, pp. 312-335, 2012.

[46] T. Liu, J. Tong, Q. Guo, J. Xi, Y. Yu, and Z. Xiao, "Energy efficiency of uplink massive MIMO systems with successive interference cancellation," IEEE Communications Letters, vol. 21, no. 3, pp. 668-671, 2016.

[47] J. Wu, Z. Chen, and M. Zhao, "SECM: status estimation and cache management algorithm in opportunistic networks," The Journal of Supercomputing, vol. 75, no. 5, pp. 2629-2647, 2019.

[48] J. Wu and Z. Chen, "Data decision and transmission based on mobile data health records on sensor devices in wireless networks," Wireless Personal Communications, vol. 90, no. 4, pp. 2073-2087, 2016. 